\title{
Monopole Floer homology \\ for rational homology 3-spheres
}

\author{
Kim A. Frøyshov
}

\begin{abstract}
We give a new construction of monopole Floer homology for $\operatorname{spin}^{c}$ rational homology 3 -spheres. As applications we define two invariants of certain 4-manifolds with $b_{1}=1$ and $b^{+}=0$.
\end{abstract}

\section{Contents}

1 Introduction

2 Main results 3

2.1 Irreducible Floer groups . . . . . . . . . . . . . . . . . 3

2.2 Equivariant Floer groups . . . . . . . . . . . . . . . . 5

2.3 The $h$-invariant . . . . . . . . . . . . . . . . . . 6

2.4 Invariants of 4 -manifolds . . . . . . . . . . . . . . . . 8

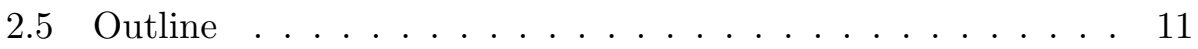

3 Chambers for the metric and perturbation

4 Moduli spaces and index $\quad \mathbf{1 7}$

5 Perturbations and reducibles $\quad 19$

6 Orientations 20

7 Irreducible Floer groups $\quad 2$

8 The $u$-map and cobordisms

9 Cobordisms and reducibles 29

10 Irreducible Floer groups for different chambers 38 


\section{Introduction}

In their recent book 13. Kronheimer and Mrowka constructed monopole Floer homology groups for arbitrary closed oriented 3-manifolds by applying a new kind of Morse homology to certain blown-up configuration spaces. In this paper we will develop the theory from a different angle in the case of an oriented rational homology 3 -sphere $Y$ equipped with a $\operatorname{spin}^{c}$ structure. Our starting-point will be the "irreducible" Floer cohomology $\operatorname{HF}^{*}(Y, m)$ which is the result of adapting Floer's original definition [6] to monopoles. Here the parameter $m$, which runs through a set $\mathfrak{m}(Y)$ of the form $m_{0}+\mathbb{Z}$ with $m_{0} \in \mathbb{Q}$, indicates which chamber for the metric and perturbation is being used. (Because of our choice of grading conventions we find it more natural to work with Floer cohomology.)

By taking suitable limits of $\operatorname{HF}^{*}(Y, m)$ as $m \rightarrow \pm \infty$ we obtain invariants $\overline{\mathrm{HF}}^{*}(Y), \underline{\mathrm{HF}}^{*}(Y)$ of the $\operatorname{spin}^{c}$ manifold $Y$. These "equivariant" Floer cohomology groups are modules over a polynomial ring and are related by a long exact sequence (which we call the fundamental sequence) involving a third module which is essentially the module of Laurent polynomials. Exact sequences of this kind are well known from Ozsváth and Szabó's Heegaard Floer homology [18] and also appear in [13. In fact, it seems likely that our equivariant groups are isomorphic to the ( $*$-versions of the) monopole Floer groups of Kronheimer and Mrowka (and their Heegaard cousins) when differences in grading conventions are taken into account.

A perhaps unusual feature in our model is that the fundamental sequence is not constructed from a short exact sequence of cochain complexes. Exactness is established by geometric means, without recourse to homological algebra.

In the case of coefficients in a field $\mathbb{F}$ of characteristic $p$ (where we allow $p=0)$ we give a precise description of how $\operatorname{HF}^{*}(Y, m ; \mathbb{F})$ depends on $m$, in terms of an invariant $h_{p}(Y) \in \mathfrak{m}(Y)$. For a fixed chamber $m$ the integer $m-h_{p}(Y)$ measures interaction between irreducible critical points and the reducible critical point of the Chern-Simons-Dirac functional, much in the 
same way as the instanton $h$-invariant (see [8]). The invariant $h_{p}(Y)$ can also be read off from the fundamental sequence.

Our approach to understanding the relationship between the groups $\operatorname{HF}^{*}(Y, m)$ for different $m$ involves a trick to avoid obstructed gluing problems. The idea is to study moduli spaces not only over the cylinder $\mathbb{R} \times Y$ but also over a manifold obtained by removing one point from $\mathbb{R} \times Y$, thereby creating an additional end $\mathbb{R}_{-} \times S^{3}$. By placing a suitable metric and perturbation on this new end we are able to correct the index of the Dirac operator. In this way we construct certain chain homomorphisms which are also essential to the proof of the exactness of the fundamental sequence.

In a slightly different direction we give some applications of Floer cohomology to $\operatorname{spin}^{c}$ smooth closed 4 -manifolds $X$ with $b_{1}(X)=1$ which contain a non-separating smoothly embedded rational homology $3-$ sphere $Y$. In the case $b^{+}(X)>1$ we express the Seiberg-Witten invariant of $X$ as the Lefschetz number of a certain endomorphism of the reduced Floer cohomology of $Y$. If $b^{+}(X)=0$ then, for certain $\operatorname{spin}^{c}$ structures on $X$, this Lefschetz number yields an invariant of $X$, i.e. is independent of $Y$. If in addition $Y$ is an integral homology sphere or $b_{2}(X)=0$ then $h_{p}(Y)$ is an invariant of $(X, e)$, where $e$ denotes the generator of $H_{3}(X ; \mathbb{Z})$ represented by $Y$.

Part of this paper was written in 2001-2 while the author was a visitor at the Institut des Hautes Études Scientifiques, to which the author is grateful for its hospitality. This work was also partially supported by NSF grant DMS-9971731, a grant from the DFG (German Research Foundation) as well as by the CRC 701 at the University of Bielefeld.

Acknowledgement: The author wishes to thank an anonymous referee for several suggestions that helped improve the exposition of the paper.

\section{Main results}

\subsection{Irreducible Floer groups}

By a $\operatorname{spin}^{c}$ manifold we shall mean a smooth, oriented manifold with a spin ${ }^{c}$ structure. The $\operatorname{spin}^{c}$ structure will usually be suppressed from notation, except in explicit computations. Let $Y$ be a $\operatorname{spin}^{c}$ rational homology 3-sphere. There is a classical invariant $\mathfrak{m}(Y) \in \mathbb{Q} / \mathbb{Z}$ which may be characterized as follows: If $X$ is any compact $\operatorname{spin}^{c} 4-$ manifold with $\partial X=Y$ (as $\operatorname{spin}^{c}$ manifolds) then

$$
\mathfrak{m}(Y) \equiv \frac{1}{8}\left(\sigma(X)-c_{1}\left(\mathcal{L}_{X}\right)^{2}\right) \quad \bmod \mathbb{Z} .
$$


Here $\mathcal{L}_{X}$ is the determinant line bundle of the $\operatorname{spin}^{c}$ structure, and $\sigma(X)$ is the signature of $X$. We will think of $\mathfrak{m}(Y)$ as a set of rational numbers of the form $m_{0}+\mathbb{Z}, m_{0} \in \mathbb{Q}$. Note that $\mathfrak{m}(Y)=\mathbb{Z}$ if $Y$ is an integral homology sphere.

For any $m \in \mathfrak{m}(Y)$ we will define an irreducible Floer cohomology group $\operatorname{HF}^{*}(Y, m)$ which is a finitely generated, graded Abelian group, where the grading runs through $2 m+\mathbb{Z}$. This is the monopole analogue of the instanton homology groups introduced by Floer [6]. We will use the set-up of [9], see in particular Section 1.2 and Chapter 3 of that book.

Choose a Riemannian metric $g$ and 1 -form $\nu$ on $Y$, and define the ChernSimons-Dirac functional $\vartheta$ as in [9, Section 3.2] with $\eta=d \nu$. Let $\mathcal{R}=$ $\mathcal{R}_{(g, \nu)}=\mathcal{R}_{Y}$ be the set of gauge equivalence classes of critical points of $\vartheta$. There is a unique reducible point in $\mathcal{R}$, which we denote by $\theta$. Set $\mathcal{R}^{*}:=\mathcal{R} \backslash\{\theta\}$. In Section 4 we will associate to any non-degenerate $\alpha \in \mathcal{R}$ an index (or degree) in $2 \mathfrak{m}(Y)+\mathbb{Z}$. For generic $\nu$, all critical points of $\vartheta$ are non-degenerate, in which case $\mathcal{R}$ is a finite set.

We can then form a cochain complex $\mathrm{CF}^{*}$ where $\mathrm{CF}^{q}$ is essentially the free Abelian group generated by the elements of $\mathcal{R}^{*}$ of index $q$, and the differential is defined in terms of Seiberg-Witten moduli spaces over the cylinder $\mathbb{R} \times Y$. The cohomology group of $\mathrm{CF}^{*}$ turns out to depend only on the $\operatorname{spin}^{c}$ manifold $Y$ and $m:=\frac{1}{2} \operatorname{ind}(\theta)$. This cohomology group is what we denote by $\operatorname{HF}^{*}(Y, m)$. It is defined for all $m \in \mathfrak{m}(Y)$ because every such $m$ can be realized for some pair $(g, \nu)$, as we will show in Section 3 .

Let $G$ be any Abelian group and $\operatorname{HF}^{*}(Y, m ; G)$ the irreducible Floer cohomology group with coefficients in $G$. If $m_{1} \leq m_{2}$ then there is a canonical homomorphism

$$
J_{m_{1}}^{m_{2}}: \operatorname{HF}^{*}\left(Y, m_{1} ; G\right) \rightarrow \operatorname{HF}^{*}\left(Y, m_{2} ; G\right)
$$

which is an isomorphism in degree $q$ unless $2 m_{1} \leq q<2 m_{2}$. These maps obey the composition law $J_{m_{2}}^{m_{3}} \circ J_{m_{1}}^{m_{2}}=J_{m_{1}}^{m_{3}}$.

Theorem 1 Let $\mathbb{F}$ be a field of characteristic $p$. Then there is an element $h_{p}(Y) \in \mathfrak{m}(Y)$ such that for Floer cohomology with coefficients in $\mathbb{F}$ the following hold:

(i) Let $m_{1} \leq m_{2} \leq h_{p}(Y)$. Then $J_{m_{1}}^{m_{2}}$ is surjective, and its kernel has dimension 1 in degree $2 m_{1}+2 k-1$ for $k=1, \ldots, m_{2}-m_{1}$ and is zero in all other degrees.

(ii) Let $h_{p}(Y) \leq m_{1} \leq m_{2}$. Then $J_{m_{1}}^{m_{2}}$ is injective, and its cokernel has dimension 1 in degree $2 m_{2}-2 k$ for $k=1, \ldots, m_{2}-m_{1}$ and is zero in all other degrees. 
A precise description of the kernel and cokernel of $J_{m_{1}}^{m_{2}}$ is provided by Proposition 13 below. Some related material can be found in [15, Section 7].

It follows from Proposition 21 below that if $\operatorname{HF}^{*}\left(Y, h_{0}(Y) ; \mathbb{Z}\right)$ is torsionfree then $\operatorname{HF}^{*}(Y, m ; \mathbb{Z})$ is torsion-free for all $m$. Now, if $Y$ admits a metric of positive scalar curvature, then $\operatorname{HF}^{*}\left(Y, h_{0}(Y) ; \mathbb{Z}\right)=0$. Applying Theorem 1 with rational coefficients then yields a complete description of $\operatorname{HF}^{*}(Y, m ; \mathbb{Z})$ for all $m$.

\subsection{Equivariant Floer groups}

We define the equivariant Floer cohomology groups $\overline{\mathrm{HF}}^{q}(Y ; G)$ and $\underline{\mathrm{HF}}^{q}(Y ; G)$ to be the direct and inverse limits, respectively, of the sequence of homomorphisms

$$
\cdots \rightarrow \operatorname{HF}^{q}(Y, m-1 ; G) \rightarrow \operatorname{HF}^{q}(Y, m ; G) \rightarrow \operatorname{HF}^{q}(Y, m+1 ; G) \rightarrow \ldots
$$

(Section 14 will provide some justification for the term "equivariant".) Clearly, there are natural homomorphisms

$$
\underline{\operatorname{HF}}^{q}(Y ; G) \rightarrow \operatorname{HF}^{q}(Y, m ; G) \rightarrow \overline{\operatorname{HF}}^{q}(Y ; G)
$$

for any $q, m$. The first of these maps is an isomorphism when $2 m \leq q$, the second one when $q \leq 2 m-1$. Hence $\underline{\operatorname{HF}}^{q}(Y ; G)$ vanishes for $q \gg 0$, and $\overline{\mathrm{HF}}^{q}(Y ; G)$ vanishes for $q \ll 0$. We define the homology groups $\overline{\mathrm{HF}}_{q}(Y ; G)$ and $\underline{\operatorname{HF}}_{q}(Y ; G)$ in a similar fashion as limits of the groups $\operatorname{HF}_{q}(Y, m ; G)$ as $m \rightarrow \mp \infty$.

The equivariant groups $\underline{\operatorname{HF}}^{*}(Y ; G)$ and $\overline{\mathrm{HF}}^{*}(Y ; G)$ both come equipped with a natural degree 2 endomorphism $u$. There is also a natural homomorphism of $\mathbb{Z}[u]$-modules

$$
J=J_{Y}: \underline{\operatorname{HF}}^{*}(Y ; G) \rightarrow \overline{\operatorname{HF}}^{*}(Y ; G)
$$

whose image we denote by $\widehat{\mathrm{HF}}^{*}(Y ; G)$ and call the reduced Floer cohomology of $Y$.

The two kinds of equivariant Floer groups are related by a long exact sequence (the fundamental sequence) which we now describe. This sequence involves the graded $\mathbb{Z}[u]$-module $P^{*}(Y)$ of "generalized polynomials"

$$
\sum_{m \in \mathfrak{m}(Y)} a_{m} x^{m}
$$


where the coefficients $a_{m}$ are integers and only finitely many of them are non-zero. The grading and module structure are given by

$$
\operatorname{deg}\left(x^{m}\right)=2 m, \quad u \cdot x^{m}=x^{m+1} .
$$

If $Y$ is an integral homology sphere then $\mathfrak{m}(Y)=\mathbb{Z}$, so in this case $P^{*}(Y)$ is the module of Laurent polynomials. There is a canonical long exact sequence of $\mathbb{Z}[u]$-homomorphisms

$$
\cdots \stackrel{D^{\prime}}{\longrightarrow} \underline{\mathrm{HF}}^{*}(Y ; G) \stackrel{J}{\longrightarrow} \overline{\mathrm{HF}}^{*}(Y ; G) \stackrel{D}{\longrightarrow} P^{*}(Y) \otimes G \stackrel{D^{\prime}}{\longrightarrow} \underline{\mathrm{HF}}^{*+1}(Y ; G) \stackrel{J}{\longrightarrow} \cdots
$$

where $J, D$ have degree 0 and $D^{\prime}$ has degree 1 . The maps $D, D^{\prime}$, which are defined at the end of Section [10, in some sense measure interaction between reducible and irreducible critical points of the Chern-Simons-Dirac functional. In our set-up this sequence is not constructed from a short exact sequence of cochain complexes. Instead, exactness is proved by means of an "almost inverse" of the map $J_{m}^{m+1}$.

Theorem 2 If $\mathbb{F}$ is any field of characteristic $p$ then $2 h_{p}(Y)$ is the lowest degree in which $D: \overline{H F}^{*}(Y ; \mathbb{F}) \rightarrow P^{*}(Y) \otimes \mathbb{F}$ is non-zero.

Thus, with field coefficients the irreducible Floer groups contain no information that cannot be read off from the sequence (11). (With integral coefficients the situation might be different.)

Recall that reversing the orientation of $Y$ reverses the sign of the ChernSimons-Dirac functional. This gives rise to canonical "Poincaré duality" isomorphisms

$$
\begin{gathered}
\operatorname{HF}^{*}(-Y,-m ; G)=\operatorname{HF}_{-1-*}(Y, m ; G), \\
\overline{\mathrm{HF}}^{*}(-Y ; G)=\underline{\mathrm{HF}_{-1-*}}(Y ; G), \quad \underline{\operatorname{HF}}^{*}(-Y ; G)=\overline{\mathrm{HF}}_{-1-*}(Y ; G),
\end{gathered}
$$

the latter two being isomorphisms of $\mathbb{Z}[u]$-modules.

\subsection{The $h$-invariant}

In this section $p$ will be fixed and we mostly write $h$ instead of $h_{p}$.

Theorem 3 For all spin ${ }^{c}$ rational homology 3-spheres $Y_{1}, Y_{2}$ one has

$$
h\left(Y_{1} \# Y_{2}\right)=h\left(Y_{1}\right)+h\left(Y_{2}\right) .
$$


Theorem 4 Let $W$ be a smooth, compact spin ${ }^{c} 4-$ manifold whose boundary is a disjoint union of rational homology spheres $Y_{1}, \ldots, Y_{r}$. If $W$ has negative definite intersection form then

$$
-\sum_{j=1}^{r} h\left(Y_{j}\right) \geq \frac{1}{8}\left(b_{2}(W)+c_{1}\left(\mathcal{L}_{W}\right)^{2}\right) .
$$

Note that if $d$ denotes the correction term in Heegaard Floer homology then the above two theorems hold with $-d / 2$ in place of $h$, see [17.

If each $Y_{j}$ is an integral homology sphere then by a theorem of Elkies [5] there is a $\operatorname{spin}^{c}$ structure on $W$ for which the right hand side of the inequality in Theorem 4 is non-negative, and positive if the intersection form is not diagonalizable over the integers.

In the case of binary polyhedral spaces, [7, Proposition 8] holds with $-8 h$ in place of $\gamma$. In particular, for the Poincaré homology sphere oriented as the boundary of the negative definite $E_{8}$-manifold one has

$$
h(\Sigma(2,3,5))=-1 .
$$

For simple lens spaces $L(q, 1), q \geq 2$, Theorem 4 and the property $h(-Y)=-h(Y)$ suffice for the computation of $h$. To see this, let $\Sigma$ be an internal connected sum of $q$ disjoint $(-1)$-spheres in the $q$-fold connected sum $W:=-q \mathbb{C P}^{2}$. Then the link of $\Sigma$, a copy of $L(q, 1)$, separates $W$ into two pieces $W_{1}, W_{2}$. Now observe that every $\operatorname{spin}^{c}$ structure on $L(q, 1)$ is the restriction of a $\operatorname{spin}^{c}$ structure $\mathfrak{s}$ on $W$ with $c_{1}(\mathfrak{s})^{2}=-q$. Applying Theorem 4 to each of $W_{1}, W_{2}$ then gives

$$
h\left(L(q, 1), \mathfrak{s}_{j}\right)=\frac{1}{8}\left[\frac{(q-2 j)^{2}}{q}-1\right]
$$

for a suitable labelling $\mathfrak{s}_{0}, \ldots, \mathfrak{s}_{q-1}$ of the $\operatorname{spin}^{c}$ structures on $L(q, 1)$.

This example shows that if $W$ is a negative definite $\operatorname{spin}^{c}$ cobordism from one rational homology 3 -sphere $Y_{1}$ to another one $Y_{2}$ (i.e. $\partial W=\left(-Y_{1}\right) \cup Y_{2}$ ) then one need not have $h\left(Y_{1}\right) \geq h\left(Y_{2}\right)$ unless $Y_{1}, Y_{2}$ are integral homology spheres.

There is a relationship between the $h$-invariant and Casson's invariant which we will now describe. It follows from Theorem 1 that $\tilde{\lambda}(Y):=$ $\chi\left(\operatorname{HF}^{*}(Y, m)\right)-m$ is independent of $m$ and therefore an invariant of the $\operatorname{spin}^{c}$ manifold $Y$. (Here $\chi$ denotes the Euler characteristic with respect to the mod 2 grading.) This result was proved with different methods by Lim [14]. He also showed that, when $Y$ is an integral homology sphere, $-\tilde{\lambda}$ agrees 
with Casson's invariant $\lambda$ (normalized so that $\lambda(\Sigma(2,3,5))=-1$ ), thereby confirming a conjecture of Kronheimer. Since $\operatorname{HF}^{*}\left(Y, h_{p}(Y) ; \mathbb{F}\right)$ maps isomorphically onto $\widehat{\mathrm{HF}}^{*}(Y ; \mathbb{F})$, we obtain:

Theorem 5 Let $\mathbb{F}$ be a field of characteristic $p$. Then for every oriented integral homology 3-sphere $Y$ one has

$$
h_{p}(Y)-\chi\left(\widehat{H F}^{*}(Y ; \mathbb{F})\right)=\lambda(Y),
$$

where $\lambda$ denotes Casson's invariant.

Similar results hold for the instanton and Heegaard Floer homologies, see [8, Section 8] and [17, Theorem 1.3].

\subsection{Invariants of $4-$ manifolds}

The results in this subsection will only be stated in their simplest form, ignoring the 1 -dimensional $\mu$-classes, and using integral coefficients for the Floer groups unless otherwise specified. Throughout the subsection $Y, Y^{\prime}$ will denote rational homology 3-spheres. All 4-manifolds will be smooth.

Let $Z$ be a compact, homology oriented $\operatorname{spin}^{c} 4-$ manifold with $\partial Z=Y$. Then $Z$ has a "relative" Seiberg-Witten invariant

$$
\bar{\psi}(Z) \in \overline{\mathrm{HF}}^{-d}(Y)
$$

where

$$
d=\frac{1}{4}\left(c_{1}\left(\mathcal{L}_{Z}\right)^{2}-\sigma(Z)\right)+b_{1}(Z)-b^{+}(Z) .
$$

If moreover $b^{+}(Z)>1$ then there is also an invariant

$$
\underline{\psi}(Z) \in \underline{\mathrm{HF}}^{-d}(Y)
$$

with $J \underline{\psi}(Z)=\bar{\psi}(Z)$.

Theorem 6 Let $Z$ be a closed, connected spin ${ }^{c}$ 4-manifold which is separated by an embedded rational homology 3-sphere $Y$ :

$$
Z=Z_{0} \cup_{Y} Z_{1}
$$

Let $Z_{0}$ and $Z_{1}$ be homology oriented and let $Z$ be equipped with the corresponding glued homology orientation as defined in [9, Section 12.6]. If $b^{+}\left(Z_{1}\right)>1$ and $\operatorname{dim} M(Z)=2 n \geq 0$, then the Seiberg-Witten invariant of $Z$ is

$$
S W(Z)=u^{n} \bar{\psi}\left(Z_{0}\right) \cdot \underline{\psi}\left(Z_{1}\right) .
$$


We now turn to cobordisms. Let $W$ be a compact, homology oriented $\operatorname{spin}^{c} 4-$ manifold with $\partial W=(-Y) \cup Y^{\prime}$. Then $W$ induces graded homomorphisms of $\mathbb{Z}[u]$-modules

$$
\underline{\psi}(W): \underline{\mathrm{HF}}^{*}(Y) \rightarrow{\underline{\mathrm{HF}^{*-d}}}^{*}\left(Y^{\prime}\right), \quad \bar{\psi}(W): \overline{\mathrm{HF}}^{*}(Y) \rightarrow \overline{\mathrm{HF}}^{*-d}\left(Y^{\prime}\right)
$$

which intertwine with the $J$-maps and therefore induce a homomorphism

$$
\hat{\psi}(W): \widehat{\mathrm{HF}}^{*}(Y) \rightarrow \widehat{\mathrm{HF}}^{*-d}\left(Y^{\prime}\right) .
$$

Here $d$ is defined as above with $W$ in place of $Z$. In general, the $\bar{\psi}$-invariant of a composite cobordism or of a composite manifold $Z \cup_{Y} W$ is the composition (or product) of the $\bar{\psi}$-invariants of the two pieces, provided the homology orientations are related as in Theorem 6. The same holds for the $\underline{\psi}$-invariants as long as these are defined. If $b^{+}(W)>0$ then $\underline{\psi}(W)$ factors through $J_{Y}$.

If $b_{1}(W)=0$ or $b^{+}(W)>0$ we define the $\mathbb{Z}[u]$-homomorphism

$$
P(W): P^{*}\left(Y_{1}\right) \rightarrow P^{*-d}\left(Y_{2}\right)
$$

as follows: If $b^{+}(W)>0$ set $P(W):=0$. If $b^{+}(W)=0=b_{1}(W)$ set $P(W) \cdot x^{m}:=x^{m-d / 2}$. Then we have a commutative diagram

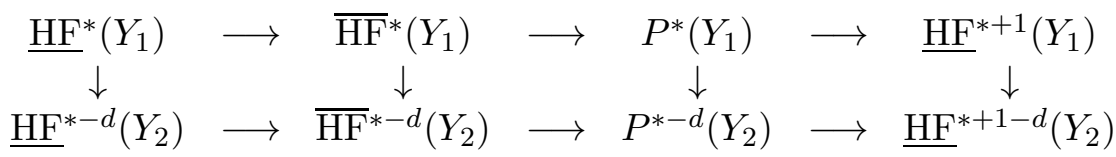

where the vertical maps are the ones induced by $W$ and the horizontal ones are as in (1). We expect that there is a similar commutative diagram when $b^{+}(W)=0, b_{1}(W)>0$ but have not yet computed the map $P(W)$ in that case. (The left-most square of the diagram commutes for any $W$, as already mentioned.)

The homomorphisms induced by cobordisms can be defined more generally for Floer groups with coefficients in any Abelian group, and have similar properties.

We will now describe an analogue of Theorem [6 for a non-separating hypersurface $Y$.

If $V=V_{0} \oplus V_{1}$ is any finitely generated mod 2 graded Abelian group then the Lefschetz number of a degree preserving endomorphism $f=f_{0} \oplus f_{1}$ of $V$ is defined as

$$
L(f):=\operatorname{tr}\left(f_{0}\right)-\operatorname{tr}\left(f_{1}\right) .
$$

If $Y$ is an oriented compact hypersurface in an oriented manifold $X$ then we denote by $X_{\| Y}$ the oriented manifold with boundary $(-Y) \cup Y$ obtained by cutting $X$ open along $Y$. 
Theorem 7 Let $X$ be a closed, connected, homology oriented spin ${ }^{c}$ 4-manifold with $b^{+}(X)>1$. Let $Y$ be a non-separating, smoothly embedded oriented rational homology 3 -sphere in $X$ and set $W=X_{\| Y}$. If $\operatorname{dim} M(X)=2 n \geq 0$ then

$$
S W(X)=L\left(u^{n} \hat{\psi}(W)\right),
$$

where $\hat{\psi}(W): \widehat{H F}^{*}(Y) \rightarrow \widehat{H F}^{*-2 n}(Y)$.

Here, as well as in Theorem 8 below, $W$ should have the homology orientation determined by the homology orientation of $X$ and the orientation of $Y$ as specified in [9, Section 12.5].

The fact that the right hand side of (3) is defined whenever $\operatorname{dim} M(X)$ is non-negative and even can be exploited to extend the Seiberg-Witten invariant to a class of 4-manifolds for which the usual definition is not available.

Theorem 8 Let $X$ be a closed, connected, homology oriented spin ${ }^{c} 4$-manifold with $b_{1}(X)=1, b^{+}(X)=0$, and $c_{1}\left(\mathcal{L}_{X}\right)^{2}=\sigma(X)$, so that $\operatorname{dim} M(X)=0$. For $j=0,1$ let $Y_{j}$ be a non-separating, smoothly embedded oriented rational homology 3-sphere in $X$. Suppose $Y_{0}$ and $Y_{1}$ represent the same generator of $H_{3}(X ; \mathbb{Z})$, and set $W_{j}=X_{\| Y_{j}}$. Then

$$
L\left(\hat{\psi}\left(W_{0}\right)\right)=L\left(\hat{\psi}\left(W_{1}\right)\right)
$$

If $Y_{0}$ and $Y_{1}$ are in fact integral homology spheres, or if $b_{2}(X)=0$, then

$$
h_{p}\left(Y_{0}\right)=h_{p}\left(Y_{1}\right)
$$

for all $p$.

Assuming the appropriate hypersurface $Y \subset X$ exists (which is not always the case) we can therefore define invariants

$$
L(X):=L(\hat{\psi}(W)), \quad h_{p}(X, e):=h_{p}(Y)
$$

where $W=X_{\| Y}$, and $e$ is the generator of $H_{3}(X ; \mathbb{Z})$ represented by $Y$. For instance, if $Y$ is any oriented integral homology 3 -sphere and $S^{1} \times Y$ has the homology orientation opposite to the one given by the canonical generators of $H^{0}\left(S^{1} \times Y\right)$ and $H^{1}\left(S^{1} \times Y\right)$ then by Theorem 5 one has

$$
L\left(S^{1} \times Y\right)=\chi\left(\widehat{\mathrm{HF}}^{*}(Y ; \mathbb{Z})\right)=\chi\left(\widehat{\mathrm{HF}}^{*}(Y ; \mathbb{Q})\right)=h_{0}(Y)-\lambda(Y) .
$$


In particular,

$$
L\left(S^{1} \times S^{3}\right)=0, \quad L\left(S^{1} \times \Sigma(2,3,7)\right)=1 .
$$

Moreover, the global spherical shell conjecture for minimal class VII surfaces with $b_{2}>0$ (known for $b_{2}=1$, see [21]) would imply that $L$ is defined and zero for such surfaces with the canonical $\operatorname{spin}^{c}$ structure.

There is an alternative description of $L(X)$ as the number of irreducible monopoles over $X$, counted with sign, for a suitable choice of metric and perturbation (using a long neck $[-T, T] \times Y$ ). However, it was shown by Okonek-Teleman [16] that this number in general depends on the chamber of the metric and perturbation.

The reader may wish to compare the definition of $L(X)$ with RubermanSaveliev's description [20] of the Furuta-Ohta invariant [10] in the case of mapping tori.

\subsection{Outline}

Here is an outline of the remainder of this paper.

Section 3 provides a proof of a technical fact which underpins our whole approach to Floer homology, namely the non-emptiness of every chamber for the metric and perturbation on a $\operatorname{spin}^{c}$ rational homology 3-sphere. The proof relies on Bär's gluing theorem for spectra of operators of Dirac type. The proof may safely be skipped on first reading, since the techniques involved are rather different from those in the rest of the paper. Section 4 introduces moduli spaces of monopoles and defines the index of a non-degenerate critical point of the Chern-Simons-Dirac functional. Section 5 shows that certain properties of reducible monopoles over 4-manifolds are stable under small perturbations. In Section 6 we specify our orientation conventions and describe the behaviour of orientations under (un)gluing maps. In Section 7 the irreducible Floer cohomology $\operatorname{HF}^{*}(Y, m)$ is defined and a first comparison of these groups for different $m$ is made. Section 8 introduces the $u$-map and establishes its compatibility with cobordisminduced homomorphisms. The subject of Section 9 is the extra structure on the irreducible Floer groups that arises from the presence of the reducible critical point, and the way this structure relates to maps induced by cobordisms. In Section 10 the trick mentioned in the introduction is used to determine the kernel and cokernel of $J_{m_{1}}^{m_{2}}$. At the end of the section the maps $D, D^{\prime}$ are defined and the exactness of the fundamental sequence ist established. In Section 11 it is shown how the reduced Floer cohomology may be computed in an arbitrary chamber for the metric and perturbation. This 
is then used to prove the Lefschetz trace formula of Theorem 7 . Section 12 contains all material on the $h$-invariant. Most of this section is logically independent from Sections 9-11. Section 13 is devoted to negative definite $4-$ manifolds with $b_{1}=1$. The final Section 14 presents a finite-dimensional analogue of the construction of the equivariant group $\overline{\mathrm{HF}}^{*}(Y)$ in order to justify the term "equivariant".

\section{Chambers for the metric and perturbation}

Let $Y$ be a $\operatorname{spin}^{c}$ rational homology 3 -sphere with Riemannian metric $g$ and perturbation 1 -form $\nu$. Let $\vartheta$ be the corresponding Chern-Simons-Dirac functional (which depends on the choice of a reference spin connection over $Y)$. The critical points of $\vartheta$ are the solutions $(B, \Psi)$ to the following 3 dimensional monopole equations:

$$
\begin{aligned}
*\left(\hat{F}_{B}+i d \nu\right) & =\frac{1}{2} \sigma(\Psi, \Psi), \\
\partial_{B} \Psi & =0
\end{aligned}
$$

Here $B$ is a spin connection over $Y$ and $\hat{F}_{B}$ half the curvature of the induced $\mathrm{U}(1)$-connection $\check{B}$, whereas $\Psi$ is a section of the spin bundle over $Y$. For more details, including the definition of the quadratic term $\sigma$, see [9, Subsection 3.2].

Any representative of the reducible critical point $\theta$ has the form $(B-i \nu, 0)$ with $\check{B}$ flat. We will say $(g, \nu)$ is a $T N D$-pair if $\theta$ is non-degenerate (i.e. if the kernel of the Dirac operator $\partial_{B-i \nu}$ is zero) and an $N D$-pair if all critical points of $\vartheta$ are non-degenerate. According to [9, Proposition 8.1.1], if $g$ is any metric on $Y$ then $(g, \nu)$ will be an ND-pair for generic $\nu$.

To any TND-pair $(g, \nu)$ we associate an index $I(g, \nu) \in \mathfrak{m}(Y)$ as follows. Let $X$ be any $\operatorname{spin}^{c}$ Riemannian 4 -manifold with one tubular end $\mathbb{R}_{+} \times Y$. Let $A$ be a spin connection over $X$ whose restriction to the end agrees with the spin connection induced by $B-i \nu$. Now set

$$
I(g, \nu)=\operatorname{ind}_{\mathbb{C}}\left(D_{A}\right)-\frac{1}{8}\left(c_{1}\left(\mathcal{L}_{X}\right)^{2}-\sigma(X)\right),
$$

where $D_{A}: L_{1}^{2}\left(X ; \mathbb{S}^{+}\right) \rightarrow L^{2}\left(X ; \mathbb{S}^{-}\right)$, cf. [9, Chapter 9]. Here $\mathbb{S}^{ \pm}$denote the spin bundles over $X$. Then $m:=I(g, \nu)$ depends only on $g, \nu$ and the orientation and $\operatorname{spin}^{c}$ structure on $Y$, essentially because the right-hand side of (4) vanishes when $X$ is closed. In situations where we are dealing 
with several pairs $(g, \nu)$ simultaneously we will often write $\theta_{m}$ and $\mathcal{R}(Y, m)$ instead of $\theta$ and $\mathcal{R}_{Y}$, resp.

The following theorem is crucial to this whole paper:

Theorem 9 For any $m \in \mathfrak{m}(Y)$ there exists a metric $g$ and 1 -form $\nu$ on $Y$ such that $m=I(g, \nu)$.

The idea of the proof, which occupies the remainder of this section, is to graft onto $Y$ a family of Berger metrics on $S^{3}$ and then apply Hitchin's computation [11] of the spectrum of the Dirac operator for these metrics together with Bär's theorem [3] on spectra of twisted Dirac operators over connected sums.

Let $\mathfrak{m}^{\prime}(Y)$ be the set of all $m \in \mathfrak{m}(Y)$ that can be realized as $I(g, \nu)$ for some pair $(g, \nu)$. Theorem 9 is an immediate consequence of the following lemma.

Lemma 1 (i) The set $\mathfrak{m}^{\prime}(Y)$ is unbounded both below and above.

(ii) If $m_{1}, m_{3} \in \mathfrak{m}^{\prime}(Y)$ and $m_{2} \in \mathfrak{m}(Y)$ satisfy $m_{1}<m_{2}<m_{3}$ then $m_{2} \in \mathfrak{m}^{\prime}(Y)$.

The proof will involve the notion of spectral flow, which for the present purposes we will interpret as an index (cf. [4, 19]). To explain this, suppose $Z$ is a closed manifold and $E \rightarrow Z$ an Hermitian vector bundle. Let $P_{t}$ : $\Gamma(E) \rightarrow \Gamma(E), a \leq t \leq b$ be a smooth family of first-order, self-adjoint, elliptic operators such that the kernels of $P_{a}$ and $P_{b}$ are zero. Choose a smooth function $f: \mathbb{R} \rightarrow[a, b]$ such that $f(t)=a$ for $t \leq-1$ and $f(t)=b$ for $t \geq 1$. We then define the spectral flow SF of the family $\left\{P_{t}\right\}_{a \leq t \leq b}$ to be the index of

$$
\frac{\partial}{\partial t}+P_{f(t)}: L_{1}^{2} \rightarrow L^{2}
$$

which is independent of $f$. (It is easy to see that $\frac{\partial}{\partial t}+P_{f(t)}$ is an elliptic operator.) We will make use of the following simple fact:

Observation 1 If $h:[a, b] \rightarrow[0, \infty)$ is a continuous function such that $\operatorname{ker}\left(P_{t}-h(t)\right)=0$ for all $t$ then $S F=n_{b}-n_{a}$, where $n_{t}$ is the number of eigenvalues of $P_{t}$ in the interval $(0, h(t))$, counted with multiplicity.

Proof of Lemma 1]: (i) Hitchin 11] computed the spectrum of the Dirac operator over $S^{3}$ for a family of metrics $\left\{g_{T}\right\}_{T>0}$ known as Berger metrics, $g_{1}$ being the standard metric. Let $D_{T}$ denote the Dirac operator over $S^{3}$ 
for the metric $g_{T}$. The eigenvalues of $D_{T}$ are given by a countable family of smooth functions of $T$, each function describing an eigenvalue of a fixed multiplicity. Each function is either $\geq \sqrt{2}$ for all $T$, or has positive derivative and one zero. Moreover, there are infinitely many functions of the second kind. The set of those $T$ for which $D_{T}$ has non-trivial kernel is a closed and discrete subset of $\mathbb{R}$ whose smallest element is 4 .

Fix $\tau>1$ with $\operatorname{ker}\left(D_{\tau}\right)=0$. The idea now is to graft the family $\left\{g_{T}\right\}_{1 \leq T \leq \tau}$ onto $Y$ and apply Bär's gluing theorem for spectra of twisted Dirac operators over connected sums [3]. To this end we fix $x_{0} \in S^{3}$ and construct a new family of metrics $g_{T, r}$ on $S^{3}$ by flattening $g_{T}$ in the geodesic ball of radius $r$ around $x_{0}$. To make this precise, choose $r_{0}>0$ such that $S^{3}$ contains a $g_{T}$-geodesic ball $U_{T}$ of radius $2 r_{0}$ around $x_{0}$ for $1 \leq T \leq \tau$. For the remainder of this paragraph we fix $T \in[1, \tau]$ and use $g_{T}$ to define exponential maps etc. Let $\bar{g}_{T}$ denote the flat metric on $U_{T}$ which under the exponential map corresponds to the constant metric $g_{T}\left(x_{0}\right)$ on $T_{x_{0}} S^{3}$. Choose a smooth function $\beta: \mathbb{R} \rightarrow \mathbb{R}$ such that $\beta(t)=0$ for $t \leq 1$ and $\beta(t)=1$ for $t \geq 2$. For $0<r<r_{0}$ define $\beta_{r}: U_{T} \rightarrow \mathbb{R}$ by

$$
\beta_{r}(\exp (v))=\beta(|v| / r)
$$

for $v \in T_{x_{0}} S^{3}$ of norm $<2 r_{0}$. Let $g_{T, r}$ be the metric on $S^{3}$ which agrees with $g_{T}$ outside $U_{T}$ and such that

$$
g_{T, r}=\beta_{r} g_{T}+\left(1-\beta_{r}\right) \bar{g}_{T} \quad \text { on } U_{T} .
$$

Then $g_{T, r}=\bar{g}_{T}$ on the ball of radius $r$ around $x_{0}$.

We will need to compare Dirac operators for different metrics on $S^{3}$. In general, if $g, \tilde{g}$ are Riemannian metrics on a spin manifold $Z$, one can identify the spin bundles such that $\tilde{g}$-Clifford multiplication with a tangent vector $e$ corresponds to $g$-Clifford multiplication with $v(e)$, where $v$ is the positive $g$-symmetric bundle automorphism of the tangent bundle $T Z$ with $v^{*} g=\tilde{g}$. Let $\nabla, \tilde{\nabla}$ be the Riemannian connections and $D, \tilde{D}$ the Dirac operators on $Z$ for the metrics $g, \tilde{g}$, resp. Then for any spinor $s$ one has a pointwise estimate

$$
|(\tilde{D}-D) s| \leq C(\alpha \cdot|\nabla s|+(1+\alpha) \cdot|\tilde{\nabla}-\nabla| \cdot|s|),
$$

where $\alpha=|\tilde{g}-g|$, norms on tensors are taken with respect to $g$, and the constant $C$ depends only on the dimension of $Z$.

Returning to our main discussion, let $D_{T, r}$ be the Dirac operator on $S^{3}$ for the metric $g_{T, r}$. It is a simple exercise to show that

$$
\sup _{1 \leq T \leq \tau}\left\|g_{T, r}-g_{T}\right\|_{C^{1}} \rightarrow 0 \quad \text { as } r \rightarrow 0^{+} .
$$


Recalling the explicit formula for the Riemannian connection in terms of the metric, we conclude that

$$
\sup _{1 \leq T \leq \tau}\left\|D_{T, r}-D_{T}\right\| \rightarrow 0 \quad \text { as } r \rightarrow 0^{+},
$$

where we use the operator norm for bounded operators $L_{1}^{2} \rightarrow L^{2}$.

We now introduce a suitable family of metrics on the cylinder $\mathbb{R} \times S^{2}$. As in [3, p 230] choose a smooth function $\rho: \mathbb{R} \rightarrow \mathbb{R}$ such that

- $\rho(t)=|t|$ for $|t| \geq 1$,

- $0<\rho(t) \leq 1$ for $|t| \leq 1$,

- $\left|\rho^{\prime}(t)\right| \leq 1$ for all $t$.

For $u>0$ set $\rho_{u}(t)=u \rho(t / u)$. Let $g_{u}^{c}$ denote the warped product metric on $\mathbb{R} \times S^{2}$ given by

$$
d s^{2}=d t^{2}+\rho_{u}(t)^{2} d \sigma^{2},
$$

where $d \sigma^{2}$ is the standard metric on $S^{2}$ and $t$ is the $\mathbb{R}$-coordinate.

Choose a metric $g$ on $Y$ for which $Y$ contains a Euclidean ball of radius $r_{0}$ around some point $y_{0}$. Fix $1 \leq T \leq \tau$ and $0<u<r<r_{0}$. In this paragraph, $S^{3}$ and $Z_{r}:=(-r, r) \times S^{2}$ will have the metrics $g_{T, r}$ and $g_{u}^{c}$, respectively, and $B_{u}(p)$ will denote the open geodesic ball of radius $u$ around a point $p$. Let $A^{ \pm}$denote the region in $Z_{r}$ defined by the inequality $u< \pm t<r$. Choose an orientation preserving isometry $\iota^{+}$between $A^{+}$ and the corresponding annulus in $Y$ around $y_{0}$. Choose a smooth family $\left\{\phi_{T}\right\}_{T>0}$ of orientation reversing orthogonal transformations $\mathbb{R}^{3} \rightarrow T_{x_{0}} S^{3}$, where $\mathbb{R}^{3}$ has the Euclidean metric. Identifying $A^{-}$with an annulus in $\mathbb{R}^{3}$ in the obvious way we obtain from $\phi_{T}$ an orientation preserving isometry $\iota^{-}$ between $A^{-}$and the corresponding annulus in $S^{3}$ around $x_{0}$. We can now introduce the glued smooth manifold

$$
Y_{T}:=S^{3} \backslash \overline{B_{u}\left(x_{0}\right)} \cup_{\iota^{-}}(-r, r) \times S^{3} \cup_{\iota^{+}} Y \backslash \overline{B_{u}\left(y_{0}\right)},
$$

which inherits a metric $\tilde{g}_{T, r, u}$ from $g_{T, r}, g_{u}^{c}, g$. Set $Y^{-}:=Y \backslash B_{r_{0}}\left(y_{0}\right)$.

Note that $Y^{-}$can also be regarded as a subset of $Y_{T}$. Since $g_{1}$ is the standard metric on $S^{3}$, there is clearly a diffeomorphism $q: Y \rightarrow Y_{1}$ which is the identity on $Y^{-}$.

The disjoint union

$$
\tilde{Y}:=\bigcup_{1 \leq T \leq \tau} Y_{T} \times\{T\}
$$


has a natural smooth structure such that the projection $\pi: \tilde{Y} \rightarrow[1, \tau]$ is a submersion. Let $\Xi$ be a vector field on $\tilde{Y}$ whose restriction to $Y^{-} \times[1, \tau]$ is $\frac{\partial}{\partial T}$ and such that $\pi_{*} \Xi=\frac{\partial}{\partial T}$. The flow of $\Xi$ together with the diffeomorphism $q: Y \rightarrow Y_{1}$ yields a diffeomorphism $Y \times[1, \tau] \rightarrow \tilde{Y}$ which commutes with the projections onto $[1, \tau]$ and is the identity on $Y^{-} \times[1, \tau]$. Let $g_{T, r, u}$ be the pull-back of the metric $\tilde{g}_{T, r, u}$ by the induced diffeomorphism $Y \rightarrow Y_{T}$.

Choose a connection in the determinant line bundle $\mathcal{L}_{0} \rightarrow Y$ which is flat outside $Y^{-}$and such that the kernel of the corresponding Dirac operator $D$ for the metric $g$ is zero (see [9, Lemma 8.1.2]). In this proof, all Dirac operators over $Y$ will be defined using this connection. Let $D_{T, r, u}$ denote the Dirac operator on $Y$ for the metric $g_{T, r, u}$. Choose $\Lambda>0$ such that neither of the operators $D_{1}$ and $D$ has any eigenvalue in $[-\Lambda, \Lambda]$. Because of (5) we can find an $r_{1} \in\left(0, r_{0}\right)$ such that for $0<r<r_{1}$ the operator $D_{1, r}$ has no eigenvalue in $[-\Lambda, \Lambda]$. By the claim on [3, p 230] and the explicit expression for $\delta$ given after that claim, there is a positive, increasing function $f$ with the following significance: Set

$$
K=\left\{(r, u) \in \mathbb{R}^{2}: 0<r<r_{1}, 0<u<f(r)\right\} .
$$

Then for $(r, u) \in K$ the kernel of $D_{1, r, u}$ is zero. Since any two points in $K$ can be joined by a smooth path in $K$, it follows that the spectral flow of the Dirac operator on $Y$ along a path of metrics from $g$ to $g_{1, r, u}$ is independent of $(r, u) \in K$.

Let $T_{1}<\cdots<T_{N}$ be the values of $T \in(1, \tau)$ for which $\operatorname{ker}\left(D_{T}\right) \neq 0$. Set $T_{0}=1, T_{N+1}=\tau, d_{k}=\operatorname{dim} \operatorname{ker}\left(D_{T_{k}}\right)$. Choose $c, \lambda$ such that

$$
0<c<\min _{0 \leq k \leq N}\left(T_{k+1}-T_{k}\right), \quad 0<\lambda \leq \Lambda,
$$

and

(a) $\operatorname{ker}\left(D_{T}-\lambda\right)=0$ if $\left|T-T_{k}\right| \leq c$ for some $k$,

(b) $D_{T_{k}-c}$ has no eigenvalue in $(0, \lambda)$,

(c) $D_{T_{k}+c}$ has exactly $d_{k}$ eigenvalues in $(0, \lambda)$, counted with multiplicity.

Of course we also have

(d) $\operatorname{ker}\left(D_{T}\right)=0$ if $1 \leq T \leq \tau$ and $\left|T-T_{k}\right| \geq c$ for all $k$.

By (5) there is an $r_{2} \in\left(0, r_{1}\right)$ such that if $0<r<r_{2}$ then (a)-(d) also hold with $D_{\bullet}, r$ in place of $D_{\bullet}$. Fix such an $r$. Another application of Bär's 
gluing theorem then shows that if $u>0$ is sufficiently small then (a)-(d) also hold with $D_{\bullet}, r, u$ in place of $D_{\bullet}$. For such $r, u$ the spectral flow of the path of operators $\left\{D_{T, r, u}\right\}_{1 \leq T \leq \tau}$ is therefore $\sum d_{k}$. (This follows from Observation 1 and the addition formula for the index [9, Corollary C.0.1].) Since this sum can be made arbitrarily large by suitable choice of $\tau$, we conclude that the set $\mathfrak{m}^{\prime}(Y)$ is unbounded above. Now recall that reversing the orientation of an odd-dimensional base-manifold reverses the sign of the Dirac operator, see [9, Section 3.1]. By replacing $g_{T}$ with $\iota^{*}\left(g_{T}\right)$ for some fixed orientation reversing diffeomorphism $\iota$ of $S^{3}$ one can therefore show in a similar way that $\mathfrak{m}^{\prime}(Y)$ is unbounded below.

(ii) If $\left(g_{0}, \nu_{0}\right),\left(g_{1}, \nu_{1}\right)$ are any TND-pairs on $Y$ then these can be connected by a smooth path $\left\{\left(g_{t}, \nu_{t}\right)\right\}_{0 \leq t \leq 1}$ of metrics and 1-forms. By essentially the same argument as in [9, Lemma 8.1.2] one can show that by replacing $\nu_{t}$ by $\nu_{t}+t(1-t) \omega$ for a generic 1 -form $\omega$ on $Y$ one can arrange that

$$
\operatorname{dim}_{\mathbb{C}} \operatorname{ker}\left(D_{\left(g_{t}, \nu_{t}\right)}\right) \leq 1 \quad \text { for } 0 \leq t \leq 1,
$$

where $D_{\left(g_{t}, \nu_{t}\right)}$ denotes the Dirac operator on $Y$ for the metric $g_{t}$ and spin connection $B-i \nu_{t}$. Using Observation 1 it is easy to see that this implies statement (ii) of the lemma.

\section{Moduli spaces and index}

As in [9, Section 1.4] let $X$ be a $\operatorname{spin}^{c}$ Riemannian 4-manifold with tubular ends $\mathbb{R}_{+} \times Y_{j}, j=1, \ldots, r$, where each $Y_{j}$ is a rational homology sphere. Let $\left(g_{j}, \nu_{j}\right)$ be an ND-pair for $Y_{j}$ as defined at the beginning of Section 3 , By a GM-pair for $X$ (with respect to the above structure) we mean a pair $(\bar{g}, \mu)$ consisting of a metric $\bar{g}$ and 2 -form $\mu$ on $X$ which restrict to $1 \times g_{j}$ resp. $\pi_{j}^{*} d \nu_{j}$ over the $j$ 'th end, where $\pi_{j}: \mathbb{R}_{+} \times Y_{j} \rightarrow Y_{j}$. We will refer to $I\left(g_{j}, \nu_{j}\right)$ as the index of $(\bar{g}, \mu)$ over the end $\mathbb{R}_{+} \times Y_{j}$.

Now suppose we are given such $\bar{g}, \mu$ as well as vectors $\vec{\alpha}=\left(\alpha_{1}, \ldots, \alpha_{r}\right)$ and $\overrightarrow{\mathfrak{p}}=\left(\mathfrak{p}_{1}, \ldots, \mathfrak{p}_{r}\right)$, where $\alpha_{j} \in \mathcal{R}_{Y_{j}}$ and $\mathfrak{p}_{j}$ is a perturbation parameter for the Seiberg-Witten equations over $\mathbb{R} \times Y$. We define the moduli space $M(X ; \vec{\alpha})=M(X ; \vec{\alpha} ; \overrightarrow{\mathfrak{p}})$ to be the space of gauge equivalence classes of solutions $(A, \Phi)$ to the Seiberg-Witten equations

$$
\begin{gathered}
\left(\hat{F}_{A}+i \mu+\operatorname{Pert}(S, \overrightarrow{\mathfrak{p}})\right)^{+}=Q(\Phi) \\
D_{A} \Phi=0
\end{gathered}
$$

such that $(A, \Phi)$ is asymptotic to $\alpha_{j}$ over the end $\mathbb{R}_{+} \times Y_{j}$. Here $A$ is a 
spin connection over $X$ and $\hat{F}_{A}$ half the curvature of the induced U(1)connection, $\Phi$ is a section of the positive spin bundle over $X$ and $Q(\Phi)$ a certain quadratic function of $\Phi$, whereas $\operatorname{Pert}(S, \overrightarrow{\mathfrak{p}})$ is a certain compactly supported perturbation, see [9, Section 3.4] for more details.

In the case $X=\mathbb{R} \times Y$ we denote by $M(\alpha, \beta)$ the moduli space over $\mathbb{R} \times Y$ with asymptotic limits $\alpha$ at $-\infty$ and $\beta$ at $\infty$.

By definition, $\operatorname{dim} M(X ; \vec{\alpha})$ is the expected dimension of the moduli space, i.e. the index of any Fredholm operator of a certain kind, see [9, p. 33]. In the case of reducible limits $\vec{\theta}=(\theta, \ldots, \theta)$ that Fredholm operator can be taken to be the one induced by $\left(-d^{*}+d^{+}\right) \oplus D_{A}$, which yields

$$
\operatorname{dim} M(X ; \vec{\theta})=-b_{0}(X)+b_{1}(X)-b^{+}(X)+2 \operatorname{ind}_{\mathbb{C}}\left(D_{A}\right) .
$$

Here ind $\mathbb{C}_{\mathbb{C}}\left(D_{A}\right)$ is as in (44). (A priori, one should consider $D_{A}$ as a map between certain $L^{p}$ rather than $L^{2}$ Sobolev-spaces, but this gives the same index.)

The addition formula for the index (see [9, Corollary C.0.1]) tells us how the expected dimension of the moduli space changes when two ends of $X$ with the same asymptotic limit are being glued. In the simplest case when $X$ has only two ends $\mathbb{R}_{ \pm} \times Y$ one has

$$
\operatorname{dim} M\left(X^{(T)}\right)=\operatorname{dim} M(X ; \alpha, \alpha)+n_{\alpha},
$$

where $X^{(T)}$ is the manifold obtained from $X$ by replacing the two ends by a neck $[-T, T] \times Y$, and $n_{\alpha}=0$ if $\alpha$ is irreducible and $n_{\alpha}=1$ otherwise. An analogous formula holds if $X$ has further ends which are not being glued.

Now let $Y$ be any $\operatorname{spin}^{c}$ rational homology 3 -sphere equipped with an ND-pair of index $m$. We will associate an index (or degree) to any $\alpha \in \mathcal{R}_{Y}$. Let $X$ be any connected $\operatorname{spin}^{c}$ Riemannian 4-manifold with one tubular end $\mathbb{R}_{+} \times Y$. We define ind $(\alpha) \in 2 \mathfrak{m}(Y)+\mathbb{Z}$ and $\operatorname{ind}_{2}(\alpha) \in \mathbb{Z} / 2 \mathbb{Z}$ by

$$
\begin{gathered}
\operatorname{dim} M(X, \alpha)=\operatorname{ind}(\alpha)+\frac{1}{4}\left(c_{1}\left(\mathcal{L}_{X}\right)^{2}-\sigma(X)\right)-n_{\alpha}+b_{1}(X)-b^{+}(X), \\
\operatorname{dim} M(X, \alpha) \equiv \operatorname{ind}_{2}(\alpha)-n_{\alpha}+b_{1}(X)-b^{+}(X) \bmod 2 .
\end{gathered}
$$

Thus, $\operatorname{ind}_{2}(\alpha) \equiv \operatorname{ind}(\alpha) \bmod 2$ if $Y$ is an integral homology sphere. Moreover, by (7) one has

$$
\operatorname{ind}(\theta)=2 m, \quad \operatorname{ind}_{2}(\theta)=0 .
$$

If $\alpha$ is irreducible then an application of the addition formula (8) yields

$$
\operatorname{ind}_{2}(\alpha) \equiv \operatorname{dim} M(\theta, \alpha) \quad \bmod 2 .
$$


The reason for our preference for Floer cohomology rather than homology is that we want ind $(\alpha)$ to appear with a positive sign in (9), in which case the Seiberg-Witten invariants of a 4-manifold with boundary lie in the Floer cohomology of the boundary.

\section{$5 \quad$ Perturbations and reducibles}

We will usually assume that $\bar{g}, \mu, \overrightarrow{\mathfrak{p}}$ have a certain generic form with regard to reducibles in $M(X ; \vec{\theta})$. To explain this, let $\bar{g}$ be given. We first take $\overrightarrow{\mathfrak{p}}=0$. If $b^{+}(X)>0$ choose $\mu$ such that $M(X ; \vec{\theta})$ is free of reducibles. If $b_{1}(X)=0=b^{+}(X)$ then $M(X ; \vec{\theta})$ contains a unique reducible point for any $\mu$, and if in addition $\operatorname{dim} M(X ; \vec{\theta}) \geq-1$ then by [9, Lemma 14.2.1] we may choose $\mu$ such that the reducible point is regular. Having chosen $\bar{g}, \mu$ we will now show that these properties of the moduli space are retained if we allow small perturbation parameters $\mathfrak{p}_{j}$.

Let $\mathfrak{P}_{j}$ be a Banach space of perturbation parameters $\mathfrak{p}_{j}$ as in [9, Section 8.2], and set $\mathfrak{P}=\mathfrak{P}_{1} \times \ldots \mathfrak{P}_{r}$.

Proposition 1 In the above situation the following hold when $\|\overrightarrow{\mathfrak{p}}\|$ is sufficiently small:

(i) If $b^{+}(X)>0$ then $M(X ; \vec{\theta})$ is free of reducibles.

(ii) If $b_{1}(X)=0=b^{+}(X)$ then $M(X ; \vec{\theta})$ contains a unique reducible point $\omega(\overrightarrow{\mathfrak{p}})$. Moreover, if $\operatorname{dim} M(X ; \vec{\theta}) \geq-1$ then this point is a regular point of the moduli space.

Proof. We prove (ii) and leave the easier part (i) to the reader. We can identify the reducible part of $\mathcal{B}(X ; \vec{\theta})$ with a configuration space $\mathcal{B}\left(\mathcal{L}_{X}\right)$ of connections in $\mathcal{L}_{X}$. Let $A_{0}$ be a connection in $\mathcal{L}_{X}$ representing $\omega(0)$. By [9. Proposition 2.3.1] any point in $\mathcal{B}\left(\mathcal{L}_{X}\right)$ has a representative $A$ satisfying $d^{*} a=0$, where $a=A-A_{0}$. Moreover, $[A]$ lies in the reducible part $M_{\overrightarrow{\mathfrak{p}}} \subset$ $\mathcal{B}\left(\mathcal{L}_{X}\right)$ of the moduli space $M(X ; \vec{\theta} ; \overrightarrow{\mathfrak{p}})$ if and only if

$$
0=\frac{1}{2} F_{A}^{+}+i \mu^{+}+\epsilon(A, \overrightarrow{\mathfrak{p}})=\frac{1}{2} d^{+} a+\epsilon(A, \overrightarrow{\mathfrak{p}}),
$$

where $\epsilon$ is a perturbation term. For $\delta>0$ set

$$
U_{\delta}=\left\{a \in L_{1}^{p, w}\left(X ; i \Lambda^{1}\right): d^{*} a=0,\|a\|_{L_{1}^{p, w}}<\delta\right\},
$$


and consider the smooth map

$$
f: U_{1} \times \mathfrak{P} \rightarrow L^{p, w}, \quad(a, \overrightarrow{\mathfrak{p}}) \mapsto \frac{1}{2} d^{+} a+\epsilon\left(A_{0}+a, \overrightarrow{\mathfrak{p}}\right) .
$$

Since $b_{1}(X)=0=b^{+}(X)$, the derivative in the first variable $D_{1} f(0,0)=d^{+}$ is an isomorphism. By the implicit function theorem there exist $\epsilon, \delta>0$ such that if $\|\overrightarrow{\mathfrak{p}}\|<\epsilon$ then $f$ has a unique zero $(a(\overrightarrow{\mathfrak{p}}), \overrightarrow{\mathfrak{p}})$ with $a(\overrightarrow{\mathfrak{p}}) \in U_{\delta}$. We will show that $\left[A_{0}+a(\overrightarrow{\mathfrak{p}})\right]$ is the only point in $M_{\overrightarrow{\mathfrak{p}}}$ when $\overrightarrow{\mathfrak{p}}$ is sufficiently small. So suppose $\{\overrightarrow{\mathfrak{p}}(n)\}$ is a sequence in $\mathfrak{P}$ converging to 0 , and $\omega_{n} \in M_{\overrightarrow{\mathfrak{p}}(n)}$ for each $n$. After passing to a subsequence we may assume that $\omega_{n}$ chainconverges. (This does not quite follow from [9, Theorem 1.3.1] since we vary the perturbation parameters, but the proof of that theorem works just as well in the present situation.) For a rational homology 3-sphere there is only one reducible point in $\tilde{\mathcal{R}}=\mathcal{R}$, so the chain-limit lies in $M_{0}$ and is therefore equal to $\left[A_{0}\right]$. By [9, Proposition 6.4.1] the sequence $\left\{\omega_{n}\right\}$ actually converges in $\mathcal{B}\left(\mathcal{L}_{X}\right)$. Now, $A_{0}+U_{1} \rightarrow \mathcal{B}\left(\mathcal{L}_{X}\right)$ is an open map, so when $n$ is sufficiently large there is a representative of $\omega_{n}$ of the form $A_{0}+a_{n}$ such that $d^{*} a_{n}=0$ and $\left\|a_{n}\right\|_{L_{1}^{p, w}} \rightarrow 0$. But then $a_{n}=a(\overrightarrow{\mathfrak{p}}(n))$ for $n \gg 0$.

\section{Orientations}

Let $Y$ be a $\operatorname{spin}^{c}$ rational homology 3 -sphere equipped with an ND-pair. By an orientation of a critical point $\alpha \in \mathcal{R}_{Y}^{*}$ we mean an orientation of $\mathcal{B}(\theta, \alpha)=\mathcal{B}(\mathbb{R} \times Y ; \theta, \alpha)$, or more precisely, a section of the orientation cover $\lambda \rightarrow \mathcal{B}(\theta, \alpha)$ constructed in [9, Section 4.2].

We will now state the conventions that we will use for orienting moduli spaces. Let $X$ be a homology oriented Riemannian $\operatorname{spin}^{c} 4$-manifold with tubular ends modelled on rational homology spheres. The canonical orientation $o_{\text {can }}$ of $\mathcal{B}(X ; \vec{\theta})$ is the one determined by the homology orientation of $X$ as in [9, Proposition 12.2.1]. Now suppose the ends of $X$ are $\mathbb{R}_{-} \times Y_{j}^{-}=\mathbb{R}_{+} \times\left(-Y_{j}^{-}\right), j=1, \ldots, r^{-}$and $\mathbb{R}_{+} \times Y_{j}^{+}, j=1, \ldots, r^{+}$. We will refer to these as "negative" and "positive" ends, respectively. Let $\alpha_{j}^{ \pm}$be a monopole over $Y_{j}^{ \pm}$which is irreducible and equipped with an orientation $o_{j}^{ \pm}$for $j=1, \ldots, \rho^{ \pm}$and reducible for $j=\rho^{ \pm}+1, \ldots, r^{ \pm}$. Let $\vec{\alpha}^{ \pm}:=\left(\alpha_{1}^{ \pm}, \ldots, \alpha_{r^{ \pm}}^{ \pm}\right)$. We give $\mathcal{B}\left(X ; \vec{\alpha}^{-}, \vec{\alpha}^{+}\right)$the orientation $o$ satisfying

$$
o_{1}^{-} \# \cdots \# o_{\rho^{-}}^{-} \# o=o_{\text {can }} \# o_{1}^{+} \# \cdots \# o_{\rho^{+}}^{+}
$$

as orientations of $\mathcal{B}\left(X ; \vec{\theta}, \vec{\alpha}^{+}\right)$, where \# is as defined in [9, Section 13.6]. Note that this definition is sensitive to the orderings of the (positive and 
negative) ends of $X$. We call $o$ the proper orientation of $\mathcal{B}\left(X ; \vec{\alpha}^{-}, \vec{\alpha}^{+}\right)$. Recall that an orientation of $\mathcal{B}\left(X ; \vec{\alpha}^{-}, \vec{\alpha}^{+}\right)$determines an orientation of the regular and irreducible part of the moduli space $M\left(X ; \vec{\alpha}^{-}, \vec{\alpha}^{+}\right)$.

If $X=\mathbb{R} \times Y$, where the negative and positive ends are the obvious ones, then we obtain orientations of $\mathcal{B}(\mathbb{R} \times Y ; \alpha, \theta)$ and $\mathcal{B}(\mathbb{R} \times Y ; \alpha, \beta)$ for all oriented $\alpha, \beta \in \mathcal{R}_{Y}^{*}$. In particular, an orientation of $\alpha \in \mathcal{R}_{Y}^{*}$ determines an orientation of the corresponding element of $\mathcal{R}_{-Y}^{*}$ (which in turn induces the original orientation of $\alpha$, essentially because either $M(\alpha, \theta)$ or $M(\theta, \alpha)$ is even-dimensional).

Returning to the general case, note that changing the status of an "irreducible" end of $X$ from negative to positive alters the orientation of $\mathcal{B}\left(X ; \vec{\alpha}^{-}, \vec{\alpha}^{+}\right)$by a sign which only depends on the parity of $b_{0}(X)+b_{1}(X)+$ $b^{+}(X)$, the mod 2 indices $\operatorname{ind}_{2}\left(\alpha_{j}^{ \pm}\right)$, and the new and old orderings of the ends of $X$.

Proposition 2 Let $X_{1}$ and $X_{2}$ be two 4-manifolds as above, both connected. Suppose $X_{1}$ has only one positive end $\mathbb{R}_{+} \times Y$, with limit $\alpha$, and that $X_{2}$ has only one negative end $\mathbb{R}_{-} \times Y$, with the same limit $\alpha$. Let $\vec{\alpha}^{ \pm}$be the limits assigned to the negative ends of $X_{1}$ and the positive ends of $X_{2}$, respectively. For $T>0$ let $X^{(T)}$ denote the glued manifold obtained from the disjoint union $X_{1} \cup X_{2}$ by replacing the two ends $\mathbb{R}_{ \pm} \times Y$ with a neck $[-T, T] \times$ $Y$. We regard $X^{(T)}$ as having the same negative (resp. positive) ends as $X_{1}$ (resp. $X_{2}$ ). Let $X^{(T)}$ have the glued homology orientation defined in [9, Section 13.6]. Let each limit $\alpha, \alpha_{j}^{ \pm}$be oriented if irreducible, and let $o_{1}, o_{2}, o^{\prime}$ denote the proper orientations of $\mathcal{B}\left(X_{1} ; \vec{\alpha}^{-}, \alpha\right), \mathcal{B}\left(X_{2} ; \alpha, \vec{\alpha}^{+}\right)$, and $\mathcal{B}\left(X^{(T)} ; \vec{\alpha}^{-}, \vec{\alpha}^{+}\right)$, respectively. Then

$$
o_{1} \# o_{2}=o^{\prime}
$$

If $\alpha$ is irreducible, then any ungluing map as in [9, Theorem 11.1.1] is an orientation preserving diffeomorphism from an open subset of $M\left(X^{(T)} ; \vec{\alpha}^{-}, \vec{\alpha}^{+}\right)$ onto an open subset of $M\left(X_{1} ; \vec{\alpha}^{-}, \alpha\right) \times M\left(X_{2} ; \alpha, \vec{\alpha}^{+}\right)$.

Proof. Equation [10 is easily deduced from the properties of the \#operation mentioned in [9, Section 13.6]. The statement about the ungluing maps then follows from [9, Theorem 13.4.1] by unraveling the definition of \#.

We call a diffeomorphism $\epsilon$-preserving (where $\epsilon= \pm 1$ ) if it preserves or reverses orientations according to the sign of $\epsilon$. 
Proposition 3 Let $X$ be a 4-manifold as above which is connected and has only one negative end $\mathbb{R}_{-} \times Y$ and only one positive end $\mathbb{R}_{+} \times Y$. Let the same oriented irreducible limit $\alpha$ be assigned to both ends. For $T>0$ let $X^{(T)}$ denote the glued manifold obtained from $X$ by replacing the two ends $\mathbb{R}_{ \pm} \times Y$ with a neck $[-T, T] \times Y$. Let $X^{(T)}$ have the glued homology orientation defined in [9, Section 13.5]. Let $\mathcal{B}(X ; \alpha, \alpha)$ have the proper orientation, and let $\tilde{o}$ denote the corresponding glued orientation of $\mathcal{B}\left(X^{(T)}\right)$ defined in [9, Section 13.4]. Then

$$
\tilde{o}=(-1)^{a b+a+b} o_{c a n},
$$

where $a=\operatorname{ind}_{2}(\alpha)$ and $b=\operatorname{dim} M\left(X^{(T)}\right)$. Any ungluing map as in [9, Theorem 11.1.1] is a $(-1)^{a b+a+b}$-preserving diffeomorphism from an open subset of $M\left(X^{(T)}\right)$ onto an open subset of $M(X ; \alpha, \alpha)$.

Proof. The sign requires a simple computation, which we omit. The statement about the ungluing map is just a special case of [9, Theorem 13.4.1].

\section{$7 \quad$ Irreducible Floer groups}

Let $Y$ be a $\operatorname{spin}^{c}$ rational homology 3 -sphere and $m \in \mathfrak{m}(Y)$. We will now define the irreducible Floer cohomology group $\operatorname{HF}^{*}(Y, m)$. By Theorem 9 and [9, Proposition 8.1.1] we can find an ND-pair $(g, \nu)$ on $Y$ of index $m$.

We first explain how to associate signs to index 1 flow-lines. Let $\alpha, \beta \in$ $\mathcal{R}:=\mathcal{R}_{(g, \nu)}$, not both reducible, and oriented if irreducible. Let $\mathbb{R}$ act on $M:=M(\alpha, \beta)$ by

$$
r \cdot \omega:=\mathcal{T}_{-r}^{*} \omega
$$

for $(r, \omega) \in \mathbb{R} \times M$, where the translation operator $\mathcal{T}_{s}$ on $\mathbb{R} \times Y$ is defined by $\mathcal{T}_{s}(t, y)=(t+s, y)$. If $\operatorname{dim} M=1$ then the elements of the orbit space

$$
\check{M}:=M / \mathbb{R}
$$

are precisely the connected components of $M$, and to each such component $\Gamma$ we associate a sign \pm 1 according as to whether the diffeomorphism $\mathbb{R} \rightarrow \Gamma$, $r \mapsto r \cdot \omega$ preserves or reverses orientation for $\omega \in \Gamma$.

Let $\mathcal{R}^{q}$ be the set of all elements of $\mathcal{R}^{*}$ of index $q$. Let $\mathrm{CF}^{q}$ be the Abelian group generated by all pairs $(\alpha, o)$ where $\alpha \in \mathcal{R}^{q}$ and $o$ is an orientation of $\alpha$, subject to the relation $(\alpha,-o)=-(\alpha, o)$. For convenience of notation, however, we will always assume that an orientation has been chosen for every element of $\mathcal{R}^{*}$, in which case $\mathrm{CF}^{q}$ can be identified with the free Abelian group generated by $\mathcal{R}^{q}$. 
We define the differential $d=d^{q}: \mathrm{CF}^{q} \rightarrow \mathrm{CF}^{q+1}$ as usual by

$$
d \alpha=\sum_{\beta}(\# \check{M}(\alpha, \beta)) \beta,
$$

where the sum is taken over all $\beta \in \mathcal{R}^{*}$ of index $q+1$. Here and elsewhere in this paper, \# denotes a signed count. Note that the differential depends on the choice of a small perturbation of the Seiberg-Witten equations over $\mathbb{R} \times Y$ as in $[9$. However, this perturbation will not be reflected in our notation.

We also need to study flow-lines running between irreducibles and the reducible. Noting that

$$
\begin{aligned}
& \operatorname{dim} M\left(\alpha, \theta_{m}\right)=2 m-1-\operatorname{ind}(\alpha), \\
& \operatorname{dim} M\left(\theta_{m}, \alpha\right)=\operatorname{ind}(\alpha)-2 m,
\end{aligned}
$$

we define, following [4], homomorphisms

$$
\begin{aligned}
& \delta: \mathrm{CF}^{2 m-2} \rightarrow \mathbb{Z}, \quad \alpha \mapsto \# \check{M}\left(\alpha, \theta_{m}\right), \\
& \delta^{\prime}: \mathbb{Z} \rightarrow \mathrm{CF}^{2 m+1}, \quad 1 \mapsto \sum_{\alpha}\left(\# \check{M}\left(\theta_{m}, \alpha\right)\right) \alpha,
\end{aligned}
$$

where the sum is taken over all $\alpha \in \mathcal{R}^{*}$ of index $2 m+1$.

Proposition $4 d \circ d=0, \delta \circ d=0, d \circ \delta^{\prime}=0$.

Proof. This follows from [9, Section 12.2] and Proposition 2,

For a fixed Abelian group $G$ let $\mathrm{HF}^{*}$ denote the cohomology of $\mathrm{CF}^{*} \otimes G$, and let

$$
\mathrm{HF}^{2 m-2} \stackrel{\delta_{0}}{\longrightarrow} G \stackrel{\delta_{0}^{\prime}}{\longrightarrow} \mathrm{HF}^{2 m+1}
$$

be the homomorphisms induced by $\delta, \delta^{\prime}$. It is convenient to extend $\delta_{0}$ to all of $\mathrm{HF}^{*}$ by setting $\delta_{0}:=0$ in degrees different from $2 m-2$, and similarly for $\delta$.

We wish to understand the dependence of the group $\mathrm{HF}^{*}$ on $(g, \nu)$. (As we will see later, this problem has very much to do with $\delta_{0}, \delta_{0}^{\prime}$.) So let $\left(g_{j}, \nu_{j}\right), j=1,2$ be two ND-pairs for $Y$ and let $m_{j}, \mathcal{R}_{j}^{*}, \mathrm{CF}_{j}, \mathrm{HF}_{j}$ be the corresponding data as introduced above. We will investigate for which $q$ the usual cobordism construction yields a homomorphism $\mathrm{HF}_{2}^{q} \rightarrow \mathrm{HF}_{1}^{q}$. Let $\bar{g}$ be a Riemannian metric and $\mu$ a two-form on $\mathbb{R} \times Y$ such that $(\bar{g}, \mu)$ agrees with $\left(1 \times g_{2}, \pi_{1}^{*}\left(d \nu_{2}\right)\right)$ in the region $(-\infty,-1] \times Y$ and agrees with $\left(1 \times g_{1}, \pi_{2}^{*}\left(d \nu_{1}\right)\right)$ in $[1, \infty) \times Y$. Let $X_{21}$ denote the manifold $\mathbb{R} \times Y$ equipped with the pair $\bar{g}, \mu$. 
Lemma 2 Let $\alpha \in \mathcal{R}_{2}^{*}$ and $\beta \in \mathcal{R}_{1}^{*}$ be critical points of index $q$. If either $q \leq 2 m_{1}$ or $q \geq 2 m_{2}-1$ then $M\left(X_{21} ; \alpha, \beta\right)$ is compact.

Proof. Since $M\left(X_{21} ; \alpha, \beta\right)$ has dimension zero, it can only fail to be compact if it contains a sequence which has a chain-limit involving some monopole of negative index. The only candidate for such monopole would be the reducible point in $M\left(X_{21} ; \theta_{m_{2}}, \theta_{m_{1}}\right)$. For such a chain-limit to exist, however, the moduli spaces $M\left(\alpha, \theta_{m_{2}}\right)$ and $M\left(\theta_{m_{1}}, \beta\right)$ would both need to have positive dimension. The lemma now follows from the dimension formulae for these spaces given above.

For any $q$ satisfying the assumptions of the previous lemma we define the homomorphism

$$
k^{q}: \mathrm{CF}_{2}^{q} \rightarrow \mathrm{CF}_{1}^{q}, \quad \alpha \mapsto \sum_{\beta}\left(\# M\left(X_{21} ; \alpha, \beta\right)\right) \beta .
$$

Lemma 3 If $q \leq 2 m_{1}-1$ or $q \geq 2 m_{2}$ then $k^{p+1} d^{p}=d^{p} k^{p}$ for $p=q-1, q$, hence $k^{q}$ induces a homomorphism

$$
K^{q}: H F_{2}^{q} \rightarrow H F_{1}^{q}
$$

Proof. Again the point is that the assumptions on $q$ prevent factorizations through the reducible monopole on $X_{21}$.

Now suppose $m_{1} \leq m_{2}$. Reversing the roles of $Y_{1}$ and $Y_{2}$ we obtain a cobordism $X_{12}$ which by the previous lemma induces a homomorphism

$$
J^{q}: \mathrm{HF}_{1}^{q} \rightarrow \mathrm{HF}_{2}^{q}
$$

for all $q$. (That $J^{q}$ is well-defined can also be seen by a dimension count.)

Proposition 5 If $q \leq 2 m_{1}-1$ or $q \geq 2 m_{2}$ then $J^{q}$ and $K^{q}$ are inverse maps.

Proof. Gluing the positive end of $X_{12}$ with the negative end of $X_{21}$ yields a cobordism $X^{(T)}$ with a band of length $2 T$ where the metric and perturbation 2 -form agrees with $\left(1 \times g_{2}, \pi_{1}^{*}\left(d \nu_{2}\right)\right)$. Let $\alpha, \beta$ be two elements of $\mathcal{R}_{1}^{*}$ of index $q$. The assumptions on $q$ mean precisely that one of the moduli spaces $M\left(X_{12} ; \alpha, \theta_{m_{2}}\right)$ and $M\left(\theta_{m_{1}}, \beta\right)$ have negative dimension. Therefore no sequence $\omega_{n} \in M\left(X^{(T(n))} ; \alpha, \beta\right)$ with $T_{n} \rightarrow \infty$ can have a chain-limit involving the reducible monopole over $X_{21}$. Consequently, no factorizations through reducible critical points may appear in such chain-limits. Thus any chain-limit must lie in $M\left(X_{12} ; \alpha, \gamma\right) \times M\left(X_{21} ; \gamma, \beta\right)$ for some $\gamma \in \mathcal{R}_{2}^{*}$ of index 
q. The gluing theorem in [9] then says that for large $T$ the endomorphism $\ell^{q}$ of $\mathrm{CF}_{1}^{q}$ induced by the cobordism $X^{(T)}$ is equal to the composite of the homomorphisms $\mathrm{CF}_{1}^{q} \rightarrow \mathrm{CF}_{2}^{q} \rightarrow \mathrm{CF}_{1}^{q}$ induced by $X_{12}$ and $X_{21}$. Considering 1 -parameter families of moduli spaces over $\mathbb{R} \times Y$ obtained by deforming the metric and perturbation form on $X^{(T)}$ to $\left(1 \times g_{1}, \pi_{1}^{*}\left(d \nu_{1}\right)\right)$ we obtain a chain-homotopy between $\ell^{q}$ and the identity. Hence $K^{q} J^{q}=\mathrm{id}$, and a similar argument proves $J^{q} K^{q}=\mathrm{id}$.

A first corollary of this proposition is that if $m_{1}=m_{2}$ then $\mathrm{HF}_{1}^{*}$ and $\mathrm{HF}_{2}^{*}$ are canonically isomorphic.

Definition 1 For any $m \in \mathfrak{m}(Y)$ let $H F^{*}(Y, m ; G)$ denote the cohomology group of the cochain complex $C F^{*} \otimes G$ defined using an ND-pair $(g, \nu)$ of index $m$.

Similarly, we define the Floer homology $\operatorname{HF}_{*}(Y, m ; G)$ as the homology of the chain complex $\operatorname{Hom}\left(\mathrm{CF}^{*}, G\right)$.

We set $\operatorname{HF}^{*}(Y, m):=\operatorname{HF}^{*}(Y, m ; \mathbb{Z})$. We will often write $\mathrm{CF}^{*}(Y, m)$ for $\mathrm{CF}^{*}$, although $\mathrm{CF}^{*}$ really depends on $g, \nu$ and not just on $m$.

Proposition 5 now gives:

Proposition 6 Let $m_{1}, m_{2} \in \mathfrak{m}(Y)$ and $m_{1} \leq m_{2}$. If either $q \leq 2 m_{1}-1$ or $q \geq 2 m_{2}$ then the natural homomorphism $H_{F}^{q}\left(Y, m_{1} ; G\right) \rightarrow H F^{q}\left(Y, m_{2} ; G\right)$ is an isomorphism.

Let $\tilde{g}$ be the round metric on $S^{3}$. Because $\tilde{g}$ has positive scalar curvature we have $\mathcal{R}_{(\tilde{g}, 0)}=\{\theta\}$. Since $I(\tilde{g}, 0)=0$ (see [9, Ch. 9]), it follows that

$$
\operatorname{HF}^{*}\left(S^{3}, 0 ; G\right)=0 \text {. }
$$

Combining this with Proposition 6 yields:

Proposition 7 If $m \geq 0$ and either $q \leq-1$ or $q \geq 2 m$ then $H F^{q}\left(S^{3}, m ; G\right)=$ 0 . The same conclusion holds if $m \leq 0$ and either $q \leq 2 m-1$ or $q \geq 0$.

\section{The $u$-map and cobordisms}

We begin by defining, in analogy with [8, Section 3], a natural degree 2 homomorphism $u: \operatorname{HF}^{q}(Y, m ; G) \rightarrow \operatorname{HF}^{q+2}(Y, m ; G)$ for $q \neq 2 m-2,2 m-1$. The constraint on the degree is related to interaction with the reducible 
critical point. Set $\mathrm{CF}^{*}:=\mathrm{CF}^{*}(Y, m)$. The $u$-map will be induced by a homomorphism

$$
v: \mathrm{CF}^{*} \rightarrow \mathrm{CF}^{*+2}
$$

defined in all degrees. Let $\alpha, \beta \in \mathcal{R}(Y, m)$ be distinct critical points, and let $\mathbb{E} \rightarrow M(\alpha, \beta)$ and $\mathbb{F} \rightarrow \mathcal{B}^{*}([-1,1] \times Y)$ be the natural complex line bundles associated to the base-point $\left(0, y_{0}\right)$. (Here we use the same convention as in [9, Section 15.1].) Let $R: M(\alpha, \beta) \rightarrow \mathcal{B}^{*}([-1,1] \times Y)$ be the natural restriction map. This is well defined because of the unique continuation property of Dirac operators (see [12]). Note that we can identify $R^{*} \mathbb{F}=\mathbb{E}$. Choose a generic section $s$ of $\mathbb{F}$ and let $\sigma$ be the induced section of $\mathbb{E}$. If $\alpha, \beta$ are irreducible and $\operatorname{ind}(\beta)-\operatorname{ind}(\alpha)=2$ then $\sigma^{-1}(0)$ is a finite set of oriented points, and we define the matrix coefficient $\langle v \alpha, \beta\rangle$ by

$$
\langle v \alpha, \beta\rangle=\# \sigma^{-1}(0) .
$$

Proposition $8 d v-v d+\delta^{\prime} \delta=0$.

Proof. This is similar to the proof of [8, Theorem 4(ii)]. Note however, that the modifications to the sections $\sigma_{j}$ made in part (I) of that proof are unnecessary, as their role in ruling out cases (b), (c) can be replaced by a transversality argument. (See Part (V), Case 1 of the proof of Lemma 4 below.) This simplifies the proof somewhat. In particular, a general unique continuation property for monopoles is not needed for the proof of the proposition. (We do not know whether this property holds for the perturbed Seiberg-Witten equations used in this paper (see [9, Section 8.2]), because these equations are not of gradient flow type - in fact the perturbations are non-local in the time direction. In particular, we do not see how to apply the unique continuation results of [13].)

Up to sign, the coefficient of $\delta^{\prime} \delta$ in the proposition is the Euler number of the rank 1 Hermitian vector bundle over $S^{2}=D^{2} \cup_{S^{1}} D^{2}$ whose "clutching map" $S^{1} \rightarrow \mathrm{U}(1)$ has degree 1 . The sign can be determined using Proposition 2 .

Definition 2 For $q \neq 2 m-2,2 m-1$ we define

$$
u: H F^{q}(Y, m ; G) \rightarrow H F^{q+2}(Y, m ; G)
$$

to be the homomorphism induced by $v$.

It will follow from Proposition 9 below that $u$ is independent of the choice of section $\sigma$ and of the metric and perturbations used in the definition of the Floer cohomology. 

by

If $W$ is any compact $\operatorname{spin}^{c} 4-$ manifold with boundary $Y$ then we denote

$$
\hat{W}:=W \cup_{Y}([0, \infty) \times Y)
$$

the result of attaching a half-infinite cylinder to each boundary component of $W$. Now suppose $Y=\left(-Y_{1}\right) \cup Y_{2}$, where each $Y_{j}$ is a rational homology 3 -sphere. If $\alpha_{j} \in \mathcal{R}\left(Y, m_{j}\right), j=1,2$, then

$$
\operatorname{dim} M\left(\hat{W} ; \alpha_{1}, \alpha_{2}\right)=\operatorname{ind}\left(\alpha_{2}\right)-\operatorname{ind}\left(\alpha_{1}\right)-n_{\alpha_{2}}+d,
$$

where

$$
d=\frac{1}{4}\left(c_{1}\left(\mathcal{L}_{W}\right)^{2}-\sigma(W)\right)+b_{1}(W)-b^{+}(W) .
$$

Let $m_{j} \in \mathfrak{m}\left(Y_{j}\right)$ and set $k=m_{2}-m_{1}+\frac{d}{2} \in \frac{1}{2} \mathbb{Z}$, so that

$$
\operatorname{dim} M\left(\hat{W} ; \theta_{m_{1}}, \theta_{m_{2}}\right)=2 k-1 .
$$

If either $b^{+}(W)>1$ or $k>-1$ then $W$ gives rise to a cochain map

$$
\begin{gathered}
W^{\#}: \mathrm{CF}^{*}\left(Y_{1}, m_{1}\right) \rightarrow \mathrm{CF}^{*-d}\left(Y_{2}, m_{2}\right), \\
\alpha \mapsto \sum_{\beta}(\# M(\hat{W} ; \alpha, \beta)) \beta,
\end{gathered}
$$

which in turn induces a homomorphism

$$
W^{*}: \operatorname{HF}^{*}\left(Y_{1}, m_{1} ; G\right) \rightarrow \operatorname{HF}^{*-d}\left(Y_{2}, m_{2} ; G\right) .
$$

Note that $W^{*}$ only depends on the $\operatorname{spin}^{c}$ manifold $W$. The constraint on $b^{+}$ or $k$ was imposed to rule out factorizations through a reducible monopole on $W$. If $b^{+}(W)>1$ then generically there is no reducible on $X$ even in a one-parameter family of metrics and 2-forms $\mu$ (cf. Proposition 1 (i)). If $k>-1$ and $\alpha, \beta$ are irreducible then for dimensional reasons no reducible monopole over $W$ can appear in the chain-limit of a sequence in $M(W ; \alpha, \beta)$ provided that moduli space has dimension $\leq 1$, and the analogous statement holds for parametrized moduli spaces of dimension $\leq 1$.

These maps $W^{*}$ are functorial with respect to composition of cobordisms as long as the composite cobordism has the glued homology orientation and also satisfies $b^{+}>1$ or $k>-1$.

If $b^{+}(W) \leq 1$ and $k \leq-1$ then $W^{*}$ can still be defined under certain restrictions on the degree, cf. Lemma 3 .

We will now show that $W^{*}$ commutes with the $u$-maps, in as far as these are defined. Let $v_{j}: \mathrm{CF}^{*}\left(Y_{j}, m_{j}\right) \rightarrow \mathrm{CF}^{*+2}\left(Y_{j}, m_{j}\right)$ be a homomorphism as above. 
Proposition 9 If $b^{+}(W)>1$ or $k \geq 0$ then there exists a homomorphism $\phi: C F^{*}\left(Y_{1}, m_{1}\right) \rightarrow C F^{*-d+1}\left(Y_{2}, m_{2}\right)$ such that

$$
W^{\#} v_{1}-v_{2} W^{\#}+\delta^{\prime} \delta_{W}+\delta_{W}^{\prime} \delta=d \phi \pm \phi d
$$

as maps $C F^{*}\left(Y_{1}, m_{1}\right) \rightarrow C F^{*-d+2}\left(Y_{2}, m_{2}\right)$, where

$$
\begin{aligned}
& \delta_{W}: C F^{2 m_{2}-1+d}\left(Y_{1}, m_{1}\right) \rightarrow \mathbb{Z}, \quad \alpha \mapsto \# M\left(\hat{W} ; \alpha, \theta_{m_{2}}\right), \\
& \delta_{W}^{\prime}: \mathbb{Z} \rightarrow C F^{2 m_{1}-d}\left(Y_{2}, m_{2}\right), \quad 1 \mapsto \sum_{\beta}\left(\# M\left(\hat{W} ; \theta_{m_{1}}, \beta\right)\right) \beta,
\end{aligned}
$$

and $\delta_{W}:=0$ in degrees different from $2 m_{2}-1+d$.

Proof. See [8, Theorem 6].

\section{Corollary 1}

$$
W^{*} u=u W^{*}: H F^{q}\left(Y_{1}, m_{1}\right) \rightarrow H F^{q-d+2}\left(Y_{2}, m_{2}\right)
$$

for all $q$ for which both $u-$ maps are defined.

The assumption that $b^{+}(W)>1$ or $k \geq 0$ is only used to rule out that a 2 -dimensional moduli space $M(\hat{W} ; \alpha, \beta)$ with $\alpha, \beta$ irreducible may contain a chain-convergent sequence whose limit involves a reducible monopole on $\hat{W}$. Therefore, if $b^{+}(X) \leq 1$ and $k<0$ then the conclusion of the proposition still holds under certain restrictions on the degree.

We will now define the $u$-maps on the equivariant Floer groups $\mathrm{HF}^{*}(Y ; G)$ and $\overline{\mathrm{HF}}^{*}(Y ; G)$ (which for brevity we will here denote by $\underline{\mathrm{HF}}^{*}$ and $\overline{\mathrm{HF}}^{*}$ ). To define these maps in degree $q$ choose $m_{1}, m_{2} \in \mathfrak{m}(Y)$ with $2 m_{1} \leq q \leq$ $2 m_{2}-3$. We define $u: \underline{\mathrm{HF}}^{q} \rightarrow \underline{\mathrm{HF}}^{q+2}$ and $u: \overline{\mathrm{HF}}^{q} \rightarrow \overline{\mathrm{HF}}^{q+2}$ by requiring that the left- and right-most squares in the following diagram commute:

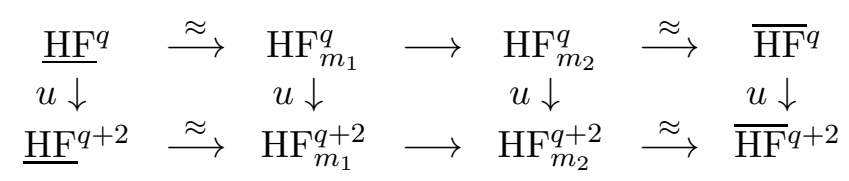

Here $\operatorname{HF}_{m}^{p}=\operatorname{HF}^{p}(Y, m ; G)$, and the horizontal maps are the natural ones. (The maps marked as isomorphisms are so by Proposition 6). This definition is independent of the choice of $m_{1}, m_{2}$. Since the middle square commutes, the homomorphism $J_{Y}: \underline{\mathrm{HF}}^{*} \rightarrow \overline{\mathrm{HF}}^{*}$ commutes with the $u$-maps. 
Let $W$ be as above, without any constraint on $b^{+}(W)$. We define $\underline{\psi}(W)$ in degree $q$ by requiring that the diagram

$$
\begin{array}{ccc}
\operatorname{HF}^{q}\left(Y_{1}, m_{1} ; G\right) & \stackrel{W^{*}}{\longrightarrow} & \operatorname{HF}^{q-d}\left(Y_{2}, m_{2} ; G\right) \\
\approx \uparrow & \approx \uparrow \\
\underline{\mathrm{HF}}^{q}\left(Y_{1} ; G\right) & \stackrel{\psi}{\longrightarrow}(W) & \underline{\mathrm{HF}}^{q-d}\left(Y_{2} ; G\right)
\end{array}
$$

commute, where $m_{1}, m_{2}$ are chosen so that $2 m_{1} \leq q$ and $2 m_{2} \leq q-d$. This definition is independent of the choice of $m_{1}, m_{2}$. In a similar fashion we define $\bar{\psi}(W)$ and the invariants $\psi(Z), \bar{\psi}(Z)$ of Subsection 2.4 when $Z$ has one boundary component.

We can now easily prove Theorem 6 by observing that when stretching $Z$ along $Y$ and using an ND-pair of index $m \gg 0$ on $Y$ then no complications with reducibles can arise.

\section{Cobordisms and reducibles}

Combining the maps $\delta_{0}, \delta_{0}^{\prime}$ of (12) with the $u$-map we obtain, for $n \geq 0$, homomorphisms

$$
\delta_{0} u^{n}: \operatorname{HF}^{2(m-n-1)}(Y, m ; G) \rightarrow G, \quad u^{n} \delta_{0}^{\prime}: G \rightarrow \operatorname{HF}^{2 m+2 n+1}(Y, m ; G)
$$

which measure interaction between reducible and irreducible critical points and will play a central role in this paper. We will now give an alternative description of these maps.

First some notation. Let $(g, \nu)$ be an ND-pair for $Y$ of index $m$ and consider the corresponding translationary invariant monopole equations on $\mathbb{R} \times Y$. Let $J$ be an open interval and $S$ a monopole over $J \times Y$ such that $\partial_{t} \vartheta\left(S_{t}\right)<0$, where $S_{t}=\left.S\right|_{\{t\} \times Y}$. (This will be the case unless $S$ is gauge equivalent to the monopole determined by some critical point of $\vartheta$, see [9, Section 7.1].) Let $\epsilon$ be a real number. If there exists a $\tau=\tau(S) \in J$ such that $[\tau-1, \tau+1] \subset J$ and

$$
\vartheta\left(S_{\tau}\right)=\vartheta\left(\theta_{m}\right)+\epsilon,
$$

then we call $\tau$ the $\epsilon$-slice of $S$.

Now set $K:=[-1,1] \times Y$ and let $\mathbb{L} \rightarrow \mathcal{B}^{*}(K)$ be the natural complex line bundle. Choose a generic smooth section $s$ of $\oplus^{n} \mathbb{L}$. Choose $\epsilon>0$ such that there is no monopole $\beta$ over $Y$ with $0<\left|\vartheta(\beta)-\vartheta\left(\theta_{m}\right)\right| \leq \epsilon$. Then for any $\alpha \in \mathcal{R}^{*}(Y, m)$ and $\omega \in M\left(\alpha, \theta_{m}\right)$ the $\epsilon$-slice $\tau(\omega)$ is well-defined. 
[9. Proposition 3.4.2 and Lemma 3.3.1] and an application of the implicit function theorem show that $\tau$ is a smooth function on $M\left(\alpha, \theta_{m}\right)$. Set

$$
M^{\prime}\left(\alpha, \theta_{m}\right):=\left\{\omega \in M\left(\alpha, \theta_{m}\right): \tau(\omega)=0, s\left(\left.\omega\right|_{K}\right)=0\right\} .
$$

If $\alpha$ has index $2(m-n-1)$ then $M^{\prime}\left(\alpha, \theta_{m}\right)$ consists of a finite number of oriented points; let $\bar{\delta}_{n}(\alpha)$ denote the number of these points counted with sign. By considering the ends of the 1 -dimensional spaces $M^{\prime}\left(\beta, \theta_{m}\right)$ where $\operatorname{ind}(\beta)=2 m-2 n-3$ one finds that $\bar{\delta}_{n} d=0$. Similarly, for any $\alpha \in \mathcal{R}^{*}(Y, m)$ and $\omega \in M\left(\theta_{m}, \alpha\right)$ the $(-\epsilon)$-slice $\tau(\omega)$ is well-defined. Setting

$$
M^{\prime}\left(\theta_{m}, \alpha\right)=\left\{\omega \in M\left(\theta_{m}, \alpha\right): \tau(\omega)=0, s\left(\left.\omega\right|_{K}\right)=0\right\}
$$

we obtain a homomorphism

$$
\bar{\delta}_{n}^{\prime}: \mathbb{Z} \rightarrow \mathrm{CF}^{2 m+2 n+1}(Y, m), \quad 1 \mapsto \sum_{\alpha}\left(\# M^{\prime}\left(\theta_{m}, \alpha\right)\right) \alpha
$$

satisfying $d \bar{\delta}_{n}^{\prime}=0$. Let

$$
\operatorname{HF}^{2(m-n-1)}(Y, m ; G) \stackrel{\delta_{n}}{\longrightarrow} G \stackrel{\delta_{n}^{\prime}}{\longrightarrow} \operatorname{HF}^{2 m+2 n+1}(Y, m ; G)
$$

be the homomorphisms induced by $\bar{\delta}_{n}, \bar{\delta}_{n}^{\prime}$. This agrees with the previous definition in the case $n=0$.

Proposition $10 \delta_{n}=\delta_{0} u^{n}$ and $\delta_{n}^{\prime}=u^{n} \delta_{0}^{\prime}$ for all $n \geq 0$.

Proof. Moving one base-point towards $-\infty$ (see the proof of Theorem 6 in [8]) one finds that $\delta_{n+1}=\delta_{n} u$. Similarly, $\delta_{n+1}^{\prime}=u \delta_{n}^{\prime}$.

We will now again consider a cobordism $W$ as in the previous section, using the same notation.

Proposition 11 If $W$ is a cobordism as in Section 8 then the following hold:

(i) If $b^{+}(W) \geq 1$ then for all $j \geq 0$,

$$
\delta_{j} W^{*}=0, \quad W^{*} \delta_{j}^{\prime}=0 .
$$

(ii) If $b_{1}(W)=b^{+}(W)=0$ and $k \geq 0$ then

$$
\begin{aligned}
& \delta_{j} W^{*}=0, \quad W^{*} \delta_{j}^{\prime}=0 \quad \text { for } 0 \leq j \leq k-1, \\
& \delta_{j} W^{*}=\delta_{j-k}, \quad W^{*} \delta_{j}^{\prime}=\delta_{j-k}^{\prime} \quad \text { for } j \geq k \text {. }
\end{aligned}
$$


Proof. We first introduce some notation that will be used in both parts of the proof. Choose a base-point $y_{j} \in Y_{j}$. We take $x_{j}:=\left(0, y_{j}\right)$ as basepoint for the band $K_{j}:=[-1,1] \times Y_{j}$. The natural complex line bundle over $\mathcal{B}^{*}\left(K_{j}\right)$ will be denoted $\mathbb{L}_{j}$. For $j=1,2$ choose $\epsilon_{j}>0$ such that there is no monopole $\beta$ over $Y_{j}$ with $0<\vartheta(\beta)-\vartheta\left(\theta_{m_{j}}\right) \leq \epsilon_{j}$. For elements $\omega$ of a moduli space $M\left(\hat{W} ; \alpha, \theta_{m_{2}}\right)$ the $\epsilon_{1}$-slice on $\mathbb{R}_{-} \times Y_{1}$ and the $\epsilon_{2}$-slice on $\mathbb{R}_{+} \times Y_{2}$ (when defined) will be denoted $\tau_{1}(\omega)$ and $\tau_{2}(\omega)$, respectively. The restriction of $\omega$ to the band $\left[\tau_{j}(\omega)-1, \tau_{j}(\omega)+1\right] \times Y_{j}$ will be denoted $R_{j}(\omega)$.

In both parts of the proof we assume that metric and perturbations on $\hat{W}$ are chosen so that the conclusions of Proposition 5 hold.

(i) By assumption there is no reducible monopole in any moduli space over $\hat{W}$. If $\epsilon_{2}$ is sufficiently small then $\tau_{2}$ is defined on any moduli space $M\left(\hat{W} ; \alpha, \theta_{m_{2}}\right)$ with $\alpha \in \mathcal{R}\left(Y_{1}, m_{1}\right)$. Let $s$ be a generic section of $\oplus^{j} \mathbb{L}_{2}$. Set

$$
M_{\alpha}^{\prime}:=\left\{\omega \in M\left(\hat{W} ; \alpha, \theta_{m_{2}}\right): s\left(R_{2}(\omega)\right)=0\right\} .
$$

If ind $(\alpha)=2 m_{2}-2 j-1+d$ then $M_{\alpha}^{\prime}$ consists of a finite number of oriented points; for irreducible $\alpha$ let $\bar{\delta}_{W, j}(\alpha)$ denote the number of these points counted with sign. If $\operatorname{ind}(\alpha)$ is one less, then since the number of ends of the 1-manifold $M_{\alpha}^{\prime}$, counted with sign, must be zero, we have

$$
\left(\bar{\delta}_{j} W^{\#}-\bar{\delta}_{W, j} d\right) \alpha=0,
$$

hence $\delta_{j} W^{*}=0$. Similarly one finds that $W^{*} \delta_{j}^{\prime}=0$.

(ii) We will focus on the statement about $\delta_{j}$. The case of $\delta_{j}^{\prime}$ is similar. We will write $\mathcal{R}_{j}=\mathcal{R}\left(Y_{j}, m_{j}\right)$ and $\mathrm{CF}_{j}^{*}=\mathrm{CF}^{*}\left(Y_{j}, m_{j}\right)$.

Let $\alpha \in \mathcal{R}_{1}^{*}$ have index $2 m_{2}-2+d=2\left(m_{1}+k\right)-2$. Counting with sign the ends of the $1-$ manifold $M\left(\hat{W} ; \alpha, \theta_{m_{2}}\right)$ we find that

$$
\left(\delta W^{\#}-\delta_{W} d\right) \alpha= \begin{cases}\delta \alpha & \text { if } k=0 \\ 0 & \text { if } k \geq 1\end{cases}
$$

If $k=0$ then $\delta_{0} W^{*}=\delta_{0}$ in cohomology, and we are done. From now on assume $k \geq 1$, in which case $\delta_{0} W^{*}=0$. Propositions 9 and 10 yield

$$
\delta_{j} W^{*}=\delta_{0} u^{j} W^{*}=\delta_{0} W^{*} u^{j}=0, \quad 0 \leq j \leq k-1 .
$$

For $j \geq k$, Proposition 9 yields

$$
\delta_{j} W^{*}=\delta_{k} u^{j-k} W^{*}=\delta_{k} W^{*} u^{j-k}, \quad j \geq k .
$$

To complete the proof of the proposition we need only prove the following lemma: 
Lemma $4 \delta_{k} W^{*}=\delta_{0}$.

Proof. Let $\alpha \in \mathcal{R}_{1}^{*}$ have index $\geq 2 m_{1}-2$. Then the moduli space $M_{\alpha}:=M\left(\hat{W} ; \alpha, \theta_{m_{2}}\right)$ has dimension $\leq 2 k+1$. We are going to cut down $M_{\alpha}$, roughly speaking, by the $k^{\prime}$ th power of the Chern class of a complex line bundle $\mathbb{L}_{\alpha}$ to obtain a manifold $\Sigma_{\alpha}$ of dimension $\leq 1$. This line bundle may be thought of as the natural line bundle associated to a base-point at $\infty$ over the end $\mathbb{R}_{+} \times Y_{2}$. (Compare the definition of $\delta_{n}$ above). The proof will involve studying the ends of $\Sigma_{\alpha}$. In the case when $\alpha$ has index $2 m_{1}-2$ special care must be taken with the part of $M_{\alpha}$ consisting of monopoles whose spinors are small over $W$. By the gluing theorem in [9], such monopoles are obtained by gluing elements of $M\left(\alpha, \theta_{m_{1}}\right):=M\left(\mathbb{R} \times Y_{1} ; \alpha, \theta_{m_{1}}\right)$ with elements of $M_{\theta}:=M\left(\hat{W} ; \theta_{m_{1}}, \theta_{m_{2}}\right)$ that are close to the reducible point $\omega_{\text {red. }}$. We will use $c_{1}\left(\mathbb{L}_{\alpha}\right)^{k-1}$ to cut down the link of $\omega_{\text {red }}$, a copy of $\mathbb{C P}^{k-1}$, to a point, while the last factor $c_{1}\left(\mathbb{L}_{\alpha}\right)$ will be played off against the gluing parameter.

Note that $M\left(\alpha, \theta_{m_{1}}\right)$ is empty if $\operatorname{ind}(\alpha)>2 m_{1}-2$ or $\vartheta(\alpha) \leq \vartheta\left(\theta_{m_{1}}\right)$. In that case

$$
\inf _{[A, \phi] \in M_{\alpha}} \int_{W}|\phi|>0
$$

and the discussion below simplifies significantly.

First some notation: Let $x_{0} \in W$ be a base-point for $W$ and $\hat{W}$. The natural complex line bundle over $M_{\alpha}$ be denoted $\mathbb{L}_{\alpha}$. As in [9] we denote by $R_{K}(S)$ or $\left.S\right|_{K}$ the restriction of a configuration $S$ (or a gauge equivalence class of such) to a codimension 0 submanifold $K \subset \hat{W}$.

(I) This part of the proof is concerned with the framed moduli space $M_{\theta, x_{0}}:=M_{x_{0}}\left(\hat{W} ; \theta_{m_{1}}, \theta_{m_{2}}\right)$ with base-point $x_{0}$. To prepare for the application of the gluing theorem we will construct the following:

- a U(1)-invariant precompact open neighbourhood $G_{0}$ of $\omega_{\text {red }}$ in $M_{\theta, x_{0}}$,

- a $\mathrm{U}(1)$-invariant open subset $V_{0} \subset \mathcal{B}_{x_{0}}(W)$ containing $R_{W}\left(\bar{G}_{0}\right)$,

- a $\mathrm{U}(1)$-equivariant smooth map $q_{0}: V_{0} \rightarrow M_{\theta, x_{0}}$ such that $q_{0}\left(\left.\omega\right|_{W}\right)=$ $\omega$ for all $\omega \in \bar{G}_{0}$.

Let $\left(A_{\text {red }}, 0\right)$ be a representative for $\omega_{\text {red }}$ and set $B_{\text {red }}:=\left.A_{\text {red }}\right|_{W}$. By [9, Section 2.5] there is a closed U(1)-invariant subspace

$$
\Xi \subset L_{1}^{p}\left(W ; i \Lambda_{W}^{1} \oplus S_{W}^{+}\right)
$$

such that

$$
\Xi^{\prime}:=\left(B_{\mathrm{red}}, 0\right)+\Xi \rightarrow \mathcal{B}_{x_{0}}(W)
$$


is a local diffeomorphism at $\left(B_{\text {red }}, 0\right)$. Since the tangent space of $M_{\theta, x_{0}}$ at $\omega_{\text {red }}$ can be identified with ker $D_{A_{\text {red }}}$, it follows by unique continuation for harmonic spinors that the restriction map

$$
R_{W}: M_{\theta, x_{0}} \rightarrow \mathcal{B}_{x_{0}}(W)
$$

is immersive at $\omega_{\text {red }}$. Let $G_{0}^{+} \subset M_{\theta, x_{0}}$ be an open subset such that

- $G_{0}^{+}$is the image of a $U(1)$-equivariant smooth embedding

$$
\iota: \mathbb{C}^{k} \rightarrow M_{\theta, x_{0}}
$$

- $R_{W}$ maps $G_{0}^{+}$diffeomorphically onto a submanifold $H \subset \mathcal{B}_{x_{0}}(W)$,

- there is a $\mathrm{U}(1)$-invariant submanifold $\tilde{H} \subset \Xi^{\prime}$ which maps diffeomorphically onto $H$.

Let $\Xi_{0} \subset \Xi$ be a closed linear complement of the tangent space $T_{\left(B_{\text {red }}, 0\right)} \tilde{H}$, so that $\Xi=T_{\left(B_{\text {red }}, 0\right)} \tilde{H} \oplus \Xi_{0}$. Then

$$
\mu: \tilde{H} \times \Xi_{0} \rightarrow \mathcal{B}_{x_{0}}(W), \quad(S, s) \mapsto[S+s]
$$

is a $\mathrm{U}(1)$-equivariant local diffeomorphism at $\left(\left(B_{\text {red }}, 0\right), 0\right)$, by the inverse function theorem. If $\pi_{1}: \tilde{H} \times \Xi_{0} \rightarrow \tilde{H}$ then $\pi_{1} \circ \mu^{-1}$ is a $\mathrm{U}(1)$-equivariant smooth map $V_{0} \rightarrow \tilde{H}$ for some $\mathrm{U}(1)$-invariant open neighbourhood $V_{0}$ of $\left.\omega_{\text {red }}\right|_{W}$. Let $\check{V}_{0}$ denote the image of $V_{0}$ in $\mathcal{B}(W)$ and $\check{V}_{0}^{*}$ the irreducible part of $\check{V}_{0}$. Let $f: \tilde{H} \rightarrow G_{0}^{+}$be the obvious map, such that $[S]=\left.f(S)\right|_{W}$ for all $S \in \tilde{H}$. Set

$$
q_{0}:=f \circ \pi_{1} \circ \mu^{-1}: V_{0} \rightarrow G_{0}^{+} \subset M_{\theta, x_{0}}
$$

and

$$
z:=\iota^{-1}: G_{0}^{+} \rightarrow \mathbb{C}^{k},
$$

and let $z_{j}$ be the $j$ 'th coordinate of $z$. If $U$ is any open subset of an unframed moduli space over $\hat{W}$ which is mapped into $\check{V}_{0}^{*}$ by $R_{W}$ then the $\mathrm{U}(1)-$ equivariant map

$$
z_{j} \circ q_{0}: V_{0} \rightarrow \mathbb{C}
$$

defines a section $\sigma_{j}$ of the appropriate complex line bundle over $U$.

Let $G_{0} \subset M_{\theta, x_{0}}$ be the image under $\iota$ of the $\rho$-ball about the origin in $\mathbb{C}^{k}$, where $\rho>0$ is small. In particular, the closure $\bar{G}_{0}$ of $G_{0}$ should be contained in the image of $q_{0}$, and $R_{W}\left(\bar{G}_{0}\right) \subset V_{0}$.

(II) This part is concerned with the moduli space $M\left(\alpha, \theta_{m_{1}}\right)$ and is only relevant in the case when ind $(\alpha)=2 m_{1}-2$ and $\vartheta(\alpha)>\vartheta\left(\theta_{m_{1}}\right)$. Then 
$M\left(\alpha, \theta_{m_{1}}\right)$ has dimension 1. Again we must construct certain data $G_{1}, V_{1}, q_{1}$ needed for the application of the gluing theorem.

For any configuration $S$ over $K_{1}$ set

$$
J(S):=\vartheta\left(S_{0}\right)
$$

where $S_{0}=\left.S\right|_{\{0\} \times Y_{1}}$. Because of the lack of unique continuation for our perturbed equations we introduce a larger band $K_{1}^{\prime}:=[-\nu, \nu] \times Y_{1}$, where $\nu \geq 1$ is chosen so large that elements of $M\left(\alpha, \theta_{m_{1}}\right)$ with $J=\vartheta\left(\theta_{m_{1}}\right)+\epsilon_{1}$ are distinguished by their restrictions to $K_{1}^{\prime}$. (The constant $\epsilon_{1}$ was chosen at the beginning of the proof of Proposition 11,) For small $\epsilon_{1}^{\prime}>0$ the set

$$
G_{1}:=\left\{\omega \in M_{x_{1}}\left(\alpha, \theta_{m_{1}}\right):\left|J(\omega)-\vartheta\left(\theta_{m_{1}}\right)-\epsilon_{1}\right|<\epsilon_{1}^{\prime}\right\}
$$

is precompact in $M_{x_{1}}\left(\alpha, \theta_{m_{1}}\right)$ and one can find a $\mathrm{U}(1)$-invariant open neighbourhood $V_{1}^{\prime}$ of $R_{K_{1}^{\prime}}\left(\bar{G}_{1}\right) \subset \mathcal{B}_{x_{1}}^{*}\left(K_{1}^{\prime}\right)$ and a $\mathrm{U}(1)$-invariant open subset $V_{1} \subset \mathcal{B}_{x_{1}}^{*}\left(K_{1}\right)$ such that

(i) $R_{K_{1}}\left(V_{1}^{\prime}\right) \subset V_{1}$,

(ii) if $\check{V}_{1} \subset \mathcal{B}\left(K_{1}\right)$ denotes the image of $V_{1}$, then $V_{1} \rightarrow \check{V}_{1}$ is a trivial $\mathrm{U}(1)-$ bundle,

(iii) for every $\omega \in \check{V}_{1}$ there exists a (necessarily unique) $\tilde{\omega} \in M\left(\alpha, \theta_{m_{1}}\right)$ with $J(\omega)=J\left(\left.\tilde{\omega}\right|_{K_{1}}\right)$,

(iv) $\check{V}_{1}^{\prime}$ is a disjoint union of open sets, one for each component of $\check{G}_{1}$ (the image of $G_{1}$ in $M\left(\alpha, \theta_{m_{1}}\right)$ ), such that $R_{K_{1}^{\prime}}$ maps each of these components into the corresponding open subset of $\check{V}_{1}^{\prime}$.

The $\tilde{\omega}$ in (iii) will be denoted $\check{q}_{1}(\omega)$. Choose a section $e$ of the bundle $V_{1} \rightarrow \check{V}_{1}$, and a section $e^{\prime}$ of the bundle $M_{x_{1}}\left(\alpha, \theta_{m_{1}}\right) \rightarrow M\left(\alpha, \theta_{m_{1}}\right)$ which agrees with the pull-back section $\left(R_{K_{1}}\right)^{*} e$ on $\check{G}_{1}$. Let $q_{1}: V_{1} \rightarrow M_{x_{1}}\left(\alpha, \theta_{m_{1}}\right)$ be the $\mathrm{U}(1)$-equivariant map satisfying $q_{1} \circ e=e^{\prime} \circ \check{q}_{1}$.

(III) We will now construct the sections of $\mathbb{L}_{\alpha}$ that will be used to define $\Sigma_{\alpha}$. For any configuration $(A, \Phi)$ over $\hat{W}$ set

$$
\ell(A, \Phi)=\int_{W}|\Phi| \cdot
$$

If $\epsilon_{0}>0$ is sufficiently small then the region $U \subset M_{\alpha}$ defined by the inequality $\ell<\epsilon_{0}$ (which is empty if $M\left(\alpha, \theta_{m_{1}}\right)$ is empty) can be described by the gluing theorem [9, Theorem 11.1.1] with $G=G_{1} \times G_{0}, K=K_{1} \amalg W$, 
$V=V_{1} \times V_{0}$, and $q=q_{1} \times q_{0}$. (Thus, roughly speaking, all elements of $U$ can be obtained by gluing elements of $G_{1}$ with elements of $G_{0}$.) We will not spell this out precisely, but contend ourselves with a list of the conditions on $\epsilon_{0}$ most relevant for the exposition (including two conditions not directly related to the gluing theorem):

(v) for any $\omega \in U$ the $\epsilon_{1}$-slice $\tau_{1}(\omega)$ on $\mathbb{R}_{-} \times Y_{1}$ is defined, $\tau_{1}(\omega)+\nu<0$, and the restriction of $\omega$ to the band $\left[\tau_{1}(\omega)-\nu, \tau_{1}(\omega)+\nu\right] \times Y_{1}$ lies in $\check{V}_{1}^{\prime}$

(vi) $\left.\omega\right|_{W} \in \check{V}_{0}$ for all $\omega \in U$,

(vii) all $\omega \in M_{\theta}$ with $\ell(\omega)<2 \epsilon_{0}$ lie in the image $\check{G}_{0}$ of $G_{0}$ in $M_{\theta}$,

(viii) $\ell>\epsilon_{0}$ on any moduli space $M\left(\hat{W} ; \beta_{1}, \beta_{2}\right)$ in which $\inf (\ell)>0$.

Note that the chain-limit (see [9, Section 7.1]) of a sequence in $U$ must either lie in $M_{\alpha}$ or have the form $\left(\omega_{1}, \omega_{0}\right)$, where $\omega_{1} \in M\left(\alpha, \theta_{m_{1}}\right)$ and $\omega_{0} \in$ $\check{G}_{0}$. The map $U \rightarrow \check{V}_{1}^{\prime}$ indicated in (v) together with the decomposition of $\check{V}_{1}^{\prime}$ in (iv) gives rise to a map $\psi: U \rightarrow \check{M}\left(\alpha, \theta_{m_{1}}\right)$.

Let $\gamma: \mathbb{R} \rightarrow \hat{W}$ be a smooth path such that $\gamma(0)=x_{0}$ and

$$
\gamma(t)= \begin{cases}\left(t, y_{1}\right) & t \leq-1 \\ \left(t, y_{2}\right) & t \geq 1\end{cases}
$$

Let $\mathcal{B}_{x}:=\mathcal{B}\left(\hat{W} ; \alpha, \theta_{m_{2}}\right)$ be the framed orbit space of configurations with base-point $x$ (see [9, Section 3.4]). For $a<b$ we define the holonomy map

$$
h_{a}^{b}: \mathcal{B}_{\gamma(a)} \rightarrow \mathcal{B}_{\gamma(b)}
$$

(depending on the choice of a reference spin connection $A_{o}$ over $\hat{W}$ ) as follows: If $S=(A, \Phi)$ is a representative of $\omega \in \mathcal{B}_{\gamma(a)}$ and $u: \hat{W} \rightarrow \mathrm{U}(1)$ an element of the group of gauge transformations acting on the configuration space $\mathcal{C}\left(\hat{W} ; \alpha, \theta_{m_{2}}\right)$ such that

$$
u(\gamma(b))=\exp \left(\int_{a}^{b} \gamma^{*}\left(A-A_{o}\right)\right)
$$

then $u(S)$ is a representative of $h_{a}^{b}(\omega)$. (Cf. [9, Eqn. 11.1].) One can easily verify that this holonomy map behaves functorially with respect to composition of paths. 
That the map $R_{1}: U \rightarrow \mathcal{B}^{*}\left(K_{1}\right)$ (defined in the very beginning of the proof of Proposition 11) is smooth follows from [9, Proposition 3.4.2 and Lemma 3.3.1] By means of holonomy along $\gamma$ the pull-back $R_{1}^{*}(e)$ defines a smooth section $\sigma^{-}$(of constant norm 1) of $\left.\mathbb{L}_{\alpha}\right|_{U}$. Because of the condition (vi) above the map (16) provides sections $\sigma_{1}, \ldots, \sigma_{k}$ of $\left.\mathbb{L}_{\alpha}\right|_{U}$. On the part of $U$ where $\sigma_{1} \neq 0$ we define a map $\eta$ into $\mathrm{U}(1)$ by the condition

$$
\sigma^{-}=\eta \cdot \sigma_{1} /\left|\sigma_{1}\right|
$$

Choose a smooth function $c: \mathbb{R} \rightarrow(1, \infty)$ such that $c(t)=t$ for $t \geq 4$ and $c(t)=2$ for $t \leq 3$. For $\omega \in M_{\alpha}$ set

$$
\bar{\tau}_{2}(\omega)= \begin{cases}c\left(\tau_{2}(\omega)\right) & \text { if } \tau_{2}(\omega) \text { is defined } \\ 2 & \text { otherwise }\end{cases}
$$

For any $\omega \in M_{\alpha}$ let $\bar{R}_{2}(\omega) \in \mathcal{B}^{*}\left(K_{2}\right)$ denote the restriction of $\omega$ to the band $\left[\bar{\tau}_{2}(\omega)-1, \bar{\tau}_{2}(\omega)+1\right] \times Y_{2}$. Choose generic smooth sections $s_{1}^{\prime}, \ldots, s_{k}^{\prime}$ of $\mathbb{L}_{2}$. By means of holonomy along $\gamma$ the pull-back $\left(\bar{R}_{2}\right)^{*} s_{j}^{\prime}$ defines a section $\sigma_{j}^{\prime}$ of $\mathbb{L}_{\alpha}$. In a similar way one obtains a section (also denoted $\sigma_{j}^{\prime}$ ) of the natural complex line bundle over $M_{\theta}^{*}$.

Choose a smooth map $\xi: \mathbb{R} \times \mathrm{U}(1) \rightarrow \mathbb{C}$ such that

$$
\xi(t, z)= \begin{cases}1, & t \geq 2 \\ z, & t \leq 1\end{cases}
$$

and such that 0 is a regular value of $\xi$. Then $\# \xi^{-1}(0)$ is independent of $\xi$, and a simple computation shows that $\# \xi^{-1}(0)=-1$.

Define a smooth section $\tilde{\sigma}_{1}$ of $\left.\mathbb{L}_{\alpha}\right|_{U}$ as follows:

$$
\tilde{\sigma}_{1}:= \begin{cases}\sigma^{-} & \text {where } \sigma_{1}=0 \\ \xi\left(-\tau_{1}\left|\sigma_{1}\right|, \eta\right) \cdot \sigma_{1} /\left|\sigma_{1}\right| & \text { where } \sigma_{1} \neq 0\end{cases}
$$

Note that if $\omega \in U$ and $\sigma_{1}(\omega)=0$ then $\tilde{\sigma}_{1}=\sigma^{-}$in a neighbourhood of $\omega$. To see this, observe that $\tau_{1}$ is bounded in any compact neighbourhood of $\omega$, hence $-\tau_{1}\left|\sigma_{1}\right| \leq 1$ in a small neighbourhood. On the other hand, if $\left|\sigma_{1}(\omega)\right| \geq 2$ then $\tilde{\sigma}_{1}=\sigma_{1} /\left|\sigma_{1}\right|$ at $\omega$, since $\tau_{1}<-1$ by definition.

Choose a smooth function $\tilde{\kappa}: \mathbb{R} \rightarrow \mathbb{R}$ such that $\tilde{\kappa}(t)=1$ for $t \geq \epsilon_{0} / 2$, $\tilde{\kappa}(t)>0$ for $t>\epsilon_{0} / 4$, and $\tilde{\kappa}(t)=0$ for $t \leq \epsilon_{0} / 4$. Set

$$
\kappa:=\tilde{\kappa} \circ \ell, \quad \underline{\kappa}:=\tilde{\kappa} \circ(\ell / 2),
$$


where $\ell$ is as in (17), and define smooth sections $\hat{\sigma}_{1}, \ldots, \hat{\sigma}_{k}$ of $\mathbb{L}_{\alpha}$ by

$$
\begin{aligned}
& \hat{\sigma}_{1}=\kappa \sigma_{1}^{\prime}+(1-\kappa) \tilde{\sigma}_{1}, \\
& \hat{\sigma}_{j}=\underline{\kappa} \sigma_{j}^{\prime}+(1-\underline{\kappa}) \sigma_{j}, \quad 2 \leq j \leq k .
\end{aligned}
$$

Note that the interpolation regions are different in the two cases: For $\hat{\sigma}_{1}$ the region is given by $\epsilon_{0} / 4<\ell<\epsilon_{0} / 2$, whereas for $\hat{\sigma}_{j}, j>1$ it is $\epsilon_{0} / 2<\ell<\epsilon_{0}$. This will be essential in the compactness argument in part (V), Case 1 below.

(IV) We will now derive a gluing result which will be used in (V). For any $\tau>0$ let $\hat{U}_{\tau}$ be the part of $U$ where $-\tau_{1}>\tau, 0<\left|\sigma_{1}\right|<\rho$, and $\sigma_{j}=0$ for $j=2, \ldots, k$. Applying the gluing theorem [9, Theorem 14.3.1] with $G=G_{1} \times G_{0}, K=K_{1} \coprod W, V=V_{1} \times V_{0}$, and $q=q_{1} \times q_{0}$ we find that if $\tau \gg 0$ then $\hat{U}_{\tau}$ is a smooth submanifold of $M_{\alpha}$ and the map

$$
\left(\eta,-\tau_{1},\left|\sigma_{1}\right|, \psi\right): \hat{U}_{\tau} \rightarrow \mathrm{U}(1) \times(\tau, \infty) \times(0, \rho) \times \check{M}\left(\alpha, \theta_{m_{1}}\right)
$$

is an orientation reversing diffeomorphism. (Argue by contradiction to prove the map is injective for $\tau \gg 0$. Another application of the gluing theorem shows that the map is also a surjective submersion for large $\tau$. To obtain submersiveness it is useful to vary the parameter $T$ in the gluing theorem as well.)

(V) Let $\Sigma_{\alpha} \subset M_{\alpha}$ be the locus where $\hat{\sigma}_{j}=0$ for $j=1, \ldots, k$. If $\epsilon_{0}>0$ is sufficiently small then $\Sigma_{\alpha}$ is transversely cut out from $M_{\alpha}$. If $\alpha$ has index $2 m_{1}-1$ then $\Sigma_{\alpha}$ consists of a finite number of points, and we define

$$
\delta_{W, k}: \mathrm{CF}_{1}^{2 m_{1}-1} \rightarrow \mathbb{Z}, \quad \beta \mapsto \# \Sigma_{\beta}
$$

Now let $\alpha$ have index $2 m_{1}-2$, in which case $\Sigma_{\alpha}$ has dimension 1 . We will determine the ends of $\Sigma_{\alpha}$. Let $\left\{\omega_{n}\right\}$ be a sequence in $\Sigma_{\alpha}$. After passing to a subsequence we may assume that $\left\{\omega_{n}\right\}$ chain-converges to some triple consisting of a broken gradient line over $\mathbb{R} \times Y_{1}$, a monopole $\omega$ over $\hat{W}$, and a broken gradient line over $\mathbb{R} \times Y_{2}$. Suppose $\omega \notin M_{\alpha}$ (or equivalently, $\omega \notin \Sigma_{\alpha}$ ).

Case 1: $\omega \in M_{\theta}$. We will show shortly that $\omega$ must be the reducible point $\omega_{\text {red. }}$. Granted this we deduce from (IV) that the corresponding number of ends of $\Sigma_{\alpha}$ is $\left(\# \xi^{-1}(0)\right) \delta \alpha=-\delta \alpha$.

Suppose now to the contrary that $\omega \neq \omega_{\text {red }}$. If $\ell(\omega)>\epsilon_{0} / 2$ then $\omega$ would satisfy the equations

$$
\begin{gathered}
\sigma_{1}^{\prime}=0, \\
\underline{\kappa} \sigma_{j}^{\prime}+(1-\underline{\kappa}) \sigma_{j}=0, \quad j=2, \ldots, k .
\end{gathered}
$$


However, the zero-set of these equations in $M_{\theta}^{*}$ is empty for a generic choice of sections $s_{j}^{\prime}$, because $\operatorname{dim} M_{\theta}^{*}=2 k-1$. Hence $\ell(\omega) \leq \epsilon_{0} / 2$, and consequently $\omega \in \check{G}_{0}$ by condition (vii) above. Certainly, $\omega$ must satisfy the equations

$$
\sigma_{j}=0, \quad j=2, \ldots, k .
$$

Therefore $\sigma_{1}(\omega) \neq 0$. Since $\tau_{1}\left(\omega_{n}\right) \rightarrow-\infty$ as $n \rightarrow \infty$, we conclude that

$$
\tilde{\sigma}_{1}\left(\omega_{n}\right)=\frac{\sigma_{1}\left(\omega_{n}\right)}{\left|\sigma_{1}\left(\omega_{n}\right)\right|} \quad \text { for } n \gg 0
$$

so $\omega$ satisfies the equation

$$
\kappa \sigma_{1}^{\prime}+\frac{1-\kappa}{\left|\sigma_{1}\right|} \sigma_{1}=0
$$

However, for dimensional reasons the common zero-set of the equations (19) and (20) in $\check{G}_{0} \backslash\left\{\omega_{\text {red }}\right\}$ is empty for a generic choice of section $s_{1}^{\prime}$. This is a contradiction, so we conclude that $\omega=\omega_{\text {red }}$ as claimed.

Case 2: $\omega \notin M_{\theta}$. Then $\ell(\omega)>\epsilon_{0}$ by condition (viii) above, so for large $n$ one has $\sigma_{j}^{\prime}\left(\omega_{n}\right)=0, j=1, \ldots, k$. There are now two possibilities: If factorization over $\mathbb{R}_{+} \times Y_{2}$ does not occur then $\bar{\tau}_{2}\left(\omega_{n}\right) \rightarrow \bar{\tau}_{2}(\omega)$ and $\omega$ will lie in some $2 k$-dimensional space $M\left(\hat{W} ; \beta_{1}, \theta_{m_{2}}\right)$ where $\beta_{1} \in \mathcal{R}_{1}^{*}$. The corresponding number of ends of $\Sigma_{\alpha}$ is $-\bar{\delta}_{W, k} d \alpha$. If such factorization does occur then $\tau_{2}\left(\omega_{n}\right) \rightarrow \infty$ and $\omega$ must lie in some 0 -dimensional space $M\left(\hat{W} ; \alpha, \beta_{2}\right)$ where $\beta_{2} \in \mathcal{R}_{2}^{*}$. The corresponding number of ends of $\Sigma_{\alpha}$ is $\bar{\delta}_{k} W^{\#} \alpha$.

Since the total number of ends of $\Sigma_{\alpha}$ must be zero, we conclude, after passing to Floer cohomology, that $\delta_{0}=\delta_{k} W^{*}$. This completes the proof of the lemma, and also of Proposition 11.

\section{Irreducible Floer groups for different chambers}

Let $Y$ be a $\operatorname{spin}^{c}$ rational homology 3 -sphere, $m \in \mathfrak{m}(Y)$, and $G$ an Abelian group. In this section we will determine the kernel and image of the canonical homomorphism

$$
\operatorname{HF}^{*}(Y, G ; m) \rightarrow \operatorname{HF}^{*}(Y, G ; m+\ell)
$$

for any natural number $\ell$. We first consider the case $\ell=1$ and then do the general case by induction on $\ell$. For $\ell=1$ Proposition 11 (ii) says that the map (21) induces a homomorphism

$$
\bar{J}: \operatorname{coker}\left(\delta_{0}^{\prime}\right) \rightarrow \operatorname{ker}\left(\delta_{0}\right) .
$$


Proposition $12 \bar{J}$ is an isomorphism.

The proof is divided into three lemmas. We begin by constructing two graded homomorphisms

$$
Q_{1}, Q_{2}: \mathrm{CF}^{*}(Y, m+1) \rightarrow \mathrm{CF}^{*}(Y, m) .
$$

such that $Q=Q_{1}+Q_{2}$ will induce an inverse of $\bar{J}$.

Fix a point $y_{0} \in Y$. Let $D \subset(-1,1) \times Y$ be a compact 4 -ball disjoint from $(-1,1) \times\left\{y_{0}\right\}$, and set $W=[-1,1] \times Y \backslash \operatorname{int}(D)$. We regard $\hat{W}$ as a manifold with two negative ends $\mathbb{R}_{-} \times Y$ and $\mathbb{R}_{-} \times S^{3}$ (in that order) and one positive end $\mathbb{R}_{+} \times Y$. The notation $M\left(\hat{W} ; \alpha_{-}, \alpha_{+}, \beta\right)$ will refer to a moduli space over $\hat{W}$ with limits $\alpha_{ \pm}$over $\mathbb{R}_{ \pm} \times Y$ and $\beta$ over $\mathbb{R}_{-} \times S^{3}$. Note that

$$
\operatorname{dim} M\left(\hat{W} ; \alpha_{-}, \alpha_{+}, \beta\right)=-\operatorname{ind}\left(\alpha_{-}\right)+\left(\operatorname{ind}\left(\alpha_{+}\right)-n_{\alpha_{+}}\right)-\operatorname{ind}(\beta) .
$$

If instead one regards $\hat{W}$ as a manifold with one negative end $\mathbb{R}_{-} \times Y$ and two positive ends $\mathbb{R}_{+} \times S^{3}$ and $\mathbb{R}_{+} \times Y$, then one obtains in general a different orientation of $M=M\left(\hat{W} ; \alpha_{-}, \alpha_{+}, \beta\right)$. If $\alpha_{+}$and $\beta$ are both

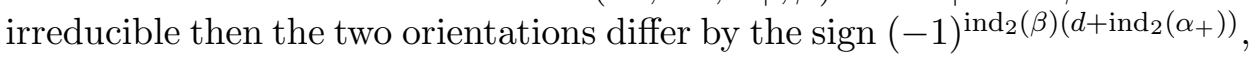
where $d \equiv \operatorname{dim} M \bmod 2$.

For the construction of $Q$ we use a GM-pair for $\hat{W}$ of index $m+1, m$, -1 on the ends $\mathbb{R}_{-} \times Y, \mathbb{R}_{+} \times Y, \mathbb{R}_{-} \times S^{3}$, resp. An important point in the proof of the proposition will be that

$$
\operatorname{dim} M\left(\hat{W} ; \theta_{m+1}, \theta_{m}, \theta_{-1}\right)=-1 .
$$

Generically, this moduli space consists of the reducible monopole $\omega_{\text {red }}$ only, and this is a regular point.

Fix a small $\epsilon>0$ and for any element $\omega$ of a moduli space $M\left(\hat{W} ; \alpha, \beta, \theta_{-1}\right)$ where $\alpha, \beta$ are not both reducible let $\tau(\omega)$ be the $(-\epsilon)$-slice over the end $\mathbb{R}_{-} \times S^{3}$, when this is defined. Let $c$ be as in (18) and define

$$
\bar{\tau}(\omega)= \begin{cases}-c(-\tau(\omega)) & \text { if } \tau(\omega) \text { is defined } \\ -2 & \text { otherwise. }\end{cases}
$$

Set $K=[-1,1] \times S^{3}$ and let $R(\omega) \in \mathcal{B}^{*}(K)$ denote the restriction of $\omega$ to the band $[\bar{\tau}(\omega)-1, \bar{\tau}(\omega)+1] \times S^{3}$. Choose a generic smooth section $s$ of the natural complex line bundle over $\mathcal{B}^{*}(K)$ and set

$$
M^{\prime}(\alpha, \beta):=\left\{\omega \in M\left(\hat{W} ; \alpha, \beta, \theta_{-1}\right): s(R(\omega))=0\right\} .
$$


If $\alpha, \beta$ are both irreducible and of the same index then $M^{\prime}(\alpha, \beta)$ is a finite set of points, so we can define $Q_{1}$ on generators $\alpha$ by

$$
Q_{1} \alpha=\sum_{\beta}\left(\# M^{\prime}(\alpha, \beta)\right) \beta
$$

According to Proposition 7 we have $\operatorname{HF}^{1}\left(S^{3},-1\right)=0$, so there is a cochain $z \in \mathrm{CF}^{0}\left(S^{3},-1\right)$ such that $\bar{\delta}_{1}^{\prime}+d z=0$. Define $Q_{2}$ by

$$
Q_{2} \alpha=\sum_{\beta}(\# M(\hat{W} ; \alpha, \beta, z)) \beta
$$

where we regard $M(\hat{W} ; \alpha, \beta, z)$ formally as a linear function in $z$. (To simplify language, we will often speak of such formal linear combinations of moduli spaces as a single moduli space.) Set $Q=Q_{1}+Q_{2}$.

Lemma $5 d Q-Q d+\delta^{\prime} \delta=0$.

Here $\delta: \mathrm{CF}^{2 m}(Y, m+1) \rightarrow \mathbb{Z}$ and $\delta^{\prime}: \mathbb{Z} \rightarrow \mathrm{CF}^{2 m+1}(Y, m)$ are the maps defined in Section 7 .

Proof. Let $\alpha \in \mathcal{R}^{*}(Y, m+1)$ and $\beta \in \mathcal{R}^{*}(Y, m)$ satisfy $\operatorname{ind}(\beta)=\operatorname{ind}(\alpha)+$ 1. Choose $\epsilon_{1}>0$ such that there is no monopole $\gamma \in \mathcal{R}^{*}(Y, m+1)$ with $0<\vartheta(\gamma)-\vartheta\left(\theta_{m+1}\right) \leq \epsilon_{1}$. Choose $\epsilon_{2}>0$ such that there is no monopole $\gamma \in \mathcal{R}^{*}(Y, m)$ with $0<\vartheta\left(\theta_{m}\right)-\vartheta(\gamma) \leq \epsilon_{2}$. For any configuration $(A, \Phi)$ over $\hat{W}$ set

$$
\ell(A, \Phi):=\int_{W}|\Phi| .
$$

Note that if $\left\{\omega_{n}\right\}$ is any chain-convergent sequence in $M(\hat{W} ; \alpha, \beta)$ with $\ell\left(\omega_{n}\right) \rightarrow 0$ then the chain-limit has the form $\left(\omega^{\prime}, \omega_{\text {red }}, \omega^{\prime \prime}\right)$, where $\omega^{\prime} \in$ $\check{M}\left(\alpha, \theta_{m+1}\right), \omega^{\prime \prime} \in \check{M}\left(\theta_{m}, \beta\right)$. Fix a small $\epsilon_{0}>0$. If $\omega \in M(\hat{W} ; \alpha, \beta)$ and $\ell(\omega)<\epsilon_{0}$ then the $\epsilon_{1}$-slice of $\omega$ on $\mathbb{R}_{-} \times Y$ and the $\epsilon_{2}$-slice of $\omega$ on $\mathbb{R}_{+} \times Y$ will be well-defined; we denote these by $\tau_{1}(\omega)$ and $\tau_{2}(\omega)$, resp. Let $R_{j}(\omega)$ denote the restriction of $\omega$ to the band $\left[\tau_{j}(\omega)-1, \tau_{j}(\omega)+1\right]$.

Let $\mathbb{L} \rightarrow M(\hat{W} ; \alpha, \beta)$ be the natural complex line bundle associated to the base-point $\left(0, y_{0}\right)$. Let $q:(-\infty, 1] \rightarrow \hat{W}$ be a path with $q(1)=\left(0, y_{0}\right) \in$ $W$ and $q(t)=\left(t, y_{-1}\right)$ for $t \leq 0$, where $y_{-1} \in S^{3}$. We use holonomy along the paths $\mathbb{R} \times\left\{y_{0}\right\}$ and $q$ to identify $\mathbb{L}$ with the natural complex line bundles associated to other base-points along these paths. Much as in the proof of Lemma 4 we construct a section $\hat{\sigma}$ of $\mathbb{L}$ such that

- $\hat{\sigma}$ agrees with the pull-back section $R^{*} s$ in the region where $\ell \geq \epsilon_{0} / 2$, 
- For $j=1,2$, if $\ell(\omega) \leq \epsilon_{0} / 4$ and $(-1)^{j}\left(\tau_{1}(\omega)+\tau_{2}(\omega)\right) \geq 1$ then $\hat{\sigma}(\omega)$ is non-zero and depends only on the restriction of $\omega$ to $\left[\tau_{j}(\omega)-1, \tau_{j}(\omega)+\right.$ 1] $\times Y$.

In the intermediate regions we interpolate as in the proof of Lemma 4. Let $\Sigma \subset M\left(\hat{W} ; \alpha, \beta, \theta_{-1}\right)$ be the zero-set of $\hat{\sigma}$.

Now let $\left\{\omega_{n}\right\}$ be a chain-convergent sequence in $\Sigma$ whose limit does not lie in $\Sigma$. If $\bar{\tau}\left(\omega_{n}\right) \rightarrow \infty$ then factorization takes place over $\mathbb{R}_{-} \times S^{3}$ through some $\gamma \in \mathcal{R}^{*}\left(S^{3},-1\right)$ of index 1 ; the corresponding number of ends of $\Sigma$ (counted with sign) is $\left\langle\chi, \bar{\delta}_{1}^{\prime}\right\rangle$, where

$$
\chi=\sum_{\gamma}(\# M(\hat{W} ; \alpha, \beta, \gamma)) \gamma \in \mathrm{CF}_{1}\left(S^{3},-1\right) .
$$

If $\bar{\tau}\left(\omega_{n}\right)$ stays bounded, then there are two cases depending on whether the chain-limit involves the reducible monopole over $\hat{W}$ or not. In the reducible case one finds as in the proof of Proposition 8 that the corresponding number of ends of $\Sigma$ is $\left\langle\delta^{\prime} \delta \alpha, \beta\right\rangle$. In the irreducible case factorization takes place over one of the tubular ends $\mathbb{R}_{ \pm} \times Y$; here the corresponding number of ends of $\Sigma$ is $\left\langle\left(d Q_{1}-Q_{1} d\right) \alpha, \beta\right\rangle$. Since the total number of ends of $\Sigma$ must be zero, we conclude that

$$
\left\langle\left(d Q_{1}-Q_{1} d+\delta^{\prime} \delta\right) \alpha, \beta\right\rangle+\left\langle\chi, \bar{\delta}_{1}^{\prime}\right\rangle=0 .
$$

Similarly, by considering the ends of $M(\hat{W} ; \alpha, \beta, z)$ we find that

$$
\left\langle\left(d Q_{2}-Q_{2} d\right) \alpha, \beta\right\rangle+\langle\chi, d z\rangle=0 .
$$

Summing the last two equations and recalling that $\bar{\delta}_{1}^{\prime}+d z=0$ we obtain

$$
\left\langle\left(d Q-Q d+\delta^{\prime} \delta\right) \alpha, \beta\right\rangle .
$$

Consider an arbitrary $m^{\prime} \in \mathfrak{m}(Y)$ and let $B: \mathrm{CF}^{*}\left(Y, m^{\prime}\right) \rightarrow \mathrm{CF}^{*}\left(Y, m^{\prime}\right)$ be the homomorphism defined in the same way as $Q$, but now using a GMpair for $\hat{W}$ of index $m^{\prime},-1$ on the ends $\mathbb{R}_{ \pm} \times Y, \mathbb{R}_{-} \times S^{3}$, resp., and with the same element $z$. Arguing as in the proof of Lemma 5 we find that $B$ is a cochain map (there are no complications from reducibles here).

Lemma $6 B$ induces the identity map on $H F^{*}\left(Y, m^{\prime} ; G\right)$.

As there are two signs in the proof which the author has not computed (and which affect the sign of $I$ in formula (23)), this lemma as well as the 
next one really hold only up to sign. However, we will ignore this, as it is irrelevant to the proof of Proposition 12.

Proof. Set $Z:=[-1,0] \times S^{3}$, and let $S_{-1}^{3}$ and $S_{0}^{3}$ be two copies of the three-sphere. We will think of $\hat{Z}$ as having tubular ends $\mathbb{R}_{-} \times S_{-1}^{3}$ and $\mathbb{R}_{+} \times S_{0}^{3}$, and the end $\mathbb{R}_{-} \times S^{3}$ of $\hat{W}$ will now be denoted $\mathbb{R}_{-} \times S_{0}^{3}$. Choose a GM-pair for $\hat{W}$ which has index $m^{\prime}$ on each of the ends $\mathbb{R}_{ \pm} \times Y$ and which agrees with $(1 \times \tilde{g}, 0)$ on $\mathbb{R}_{-} \times S_{0}^{3}$, where $\tilde{g}$ is the round metric on the threesphere. Choose a GM-pair for $\hat{Z}$ which has index -1 on the end $\mathbb{R}_{-} \times S_{-1}^{3}$ and which agrees with $(1 \times \tilde{g}, 0)$ on $\mathbb{R}_{+} \times S_{0}^{3}$. Let $X^{(T)}$ denote the manifold with tubular ends obtained from $\hat{W} \cup \hat{Z}$ by replacing the ends $\mathbb{R}_{ \pm} \times S_{0}^{3}$ with a neck $[-T, T] \times S_{0}^{3}$. Let $X^{(T)}$ be equipped with the GM-pair inherited from the GM-pairs of $\hat{W}, \hat{Z}$ in the natural way.

Now fix $\alpha, \beta \in \mathcal{R}^{*}\left(Y, m^{\prime}\right)$ of the same index and for any $\gamma \in \mathcal{R}\left(S^{3},-1\right)$ define the parametrized moduli space

$$
\mathcal{M}_{\gamma}:=\bigcup_{T \geq 1} M\left(X^{(T)} ; \alpha, \beta, \gamma\right) \times\{T\}
$$

Set $\mathcal{M}:=\mathcal{M}_{\theta_{-1}}$. Fix a small $\epsilon_{0}>0$ and let $U$ be the set of all $(\omega, T) \in \mathcal{M}$ with $\ell(\omega)<\epsilon_{0}$, where

$$
\ell(A, \Phi):=\int_{Z}|\Phi|
$$

We now copy the construction in the proof of Lemma 4 (with $k=1$ ) of a section $\hat{\sigma}_{1}$ of the natural complex line bundle over $\mathcal{M}$ corresponding to some base-point in $Z$. Here the present $Z, M\left(\hat{W} ; \alpha, \beta, \theta_{0}\right),-T$ correspond to the $W, \check{M}\left(\alpha, \theta_{m_{1}}\right), \tau_{1}$ resp. in the proof of Lemma 4. Let $\mathcal{M}^{\prime} \subset \mathcal{M}$ be the zero-set of $\hat{\sigma}_{1}$ and set

$$
\mathcal{M}^{\prime \prime}:=\mathcal{M}^{\prime}+\mathcal{M}_{z}
$$

regarded as a formal linear combination of oriented, smooth 1-manifolds with boundary. With the orientation conventions in [9] one has

$$
\# \partial \mathcal{M}^{\prime \prime}=-\langle B \alpha, \beta\rangle \text {. }
$$

We will now describe the ends of $\mathcal{M}^{\prime \prime}$. Because $\operatorname{dim} M\left(\hat{Z} ; z, \theta_{0}\right)=-1$ and there are no non-empty moduli spaces over $\hat{Z}$ of negative expected dimension, it follows that $M\left(X^{(T)} ; \alpha, \beta, z\right)$ is empty for large $T$. Therefore the ends of $\mathcal{M}_{z}$ correspond to factorization through irreducible critical points over the tubular ends $\mathbb{R}_{ \pm} \times Y$ or $\mathbb{R}_{-} \times S_{-1}^{3}$, which may occur for finitely may values of $T$. 
Determining the ends of $\mathcal{M}^{\prime}$ requires a little more consideration. Let $\left\{\left(\omega_{n}, T_{n}\right)\right\}$ be a sequence in $\mathcal{M}^{\prime}$. By passing to a subsequence we may arrange that the sequence chain-converges. This means in particular that $\left\{T_{n}\right\}$ converges to some point $T_{\infty} \in[1, \infty]$. Suppose the chain-limit does not lie in $\mathcal{M}$ (or equivalently, not in $\mathcal{M}^{\prime}$ ). We consider first the case $T_{\infty}<\infty$. Observe that

$$
\operatorname{dim} M\left(X^{\left(T_{\infty}\right)} ; \theta_{m^{\prime}}, \theta_{m^{\prime}}, \theta_{-1}\right)=1 .
$$

Therefore, for dimensional reasons the limit over $X^{\left(T_{\infty}\right)}$ cannot be reducible. Hence factorization through an irreducible critical point has taken place over $\mathbb{R}_{ \pm} \times Y$ or $\mathbb{R}_{-} \times S_{-1}^{3}$. We now turn to the case $T_{\infty}=\infty$. Since

$$
\operatorname{dim} M\left(\hat{Z} ; \theta_{-1}, \theta_{0}\right)=1
$$

the chain-limit must have the form $\left(\omega, \omega^{\prime}\right)$, where $\omega \in M\left(\hat{W} ; \alpha, \beta, \theta_{0}\right)$ and $\omega^{\prime} \in M\left(\hat{Z} ; \theta_{-1}, \theta_{0}\right)$. As in the proof of Lemma 4 (Part (V), Case 1$)$ one finds that $\omega^{\prime}$ must be the reducible point and that the corresponding number $N$ of ends of $\mathcal{M}^{\prime}$ is equal to $\# M\left(\hat{W} ; \alpha, \beta, \theta_{0}\right)$. Let $W^{(T)}$ be the result of capping off the end $\mathbb{R}_{-} \times S_{0}^{3} \subset \hat{W}$ with a disk, allowing for a long neck $[-T, T] \times S_{0}^{3}$. An easy version of the argument in [9, Part 3] yields

$$
\# M\left(\hat{W} ; \alpha, \beta, \theta_{0}\right)=\# M\left(W^{(T)} ; \alpha, \beta\right) .
$$

Deforming the metric on $W^{(T)}$ back to a cylindrical one we conclude that

$$
N=\left\langle\left(I+f_{2} d \pm d f_{2}\right) \alpha, \beta\right\rangle
$$

for some homomorphism $f_{2}: \mathrm{CF}^{*}\left(Y, m^{\prime}\right) \rightarrow \mathrm{CF}^{*-1}\left(Y, m^{\prime}\right)$.

Summing up, recall that the number of boundary points plus the number of ends of $\mathcal{M}^{\prime \prime}$ must be zero, and note that the number of ends resulting from factorizations over $\mathbb{R}_{-} \times S_{-1}^{3}$ cancel out as in the proof of Lemma 5 , It follows that

$$
\langle(-B+I+f d \pm d f) \alpha, \beta\rangle=0,
$$

where $f$ is the sum of $f_{2}$ and a homomorphism of degree -1 related to factorizations over $\mathbb{R}_{ \pm} \times Y$.

Lemma 5 shows that $Q$ induces a homomorphism

$$
\bar{Q}: \operatorname{ker}\left(\delta_{0}\right) \rightarrow \operatorname{coker}\left(\delta_{0}^{\prime}\right) .
$$

Lemma $7 \bar{J}$ and $\bar{Q}$ are inverse maps. 
Proof. We show that $\bar{J} \bar{Q}=\mathrm{id}$, the proof of $\bar{Q} \bar{J}=\mathrm{id}$ being similar. Set $Z:=[0,1] \times Y$. Choose a GM-pair for $\hat{W}$ which has index $m+1, m,-1$ over the ends $\mathbb{R}_{-} \times Y, \mathbb{R}_{+} \times Y, \mathbb{R}_{-} \times S^{3}$, resp., and a GM-pair for $\hat{Z}$ which has index $m, m+1$ over the ends $\mathbb{R}_{-} \times Y, \mathbb{R}_{+} \times Y$, resp., and such that over the ends $\mathbb{R}_{+} \times Y \subset \hat{W}$ and $\mathbb{R}_{-} \times Y \subset \hat{Z}$ these are induced from the same ND-pair for $Y$. Let $X^{(T)}$ denote the manifold with tubular ends obtained from $\hat{W} \cup \hat{Z}$ by replacing the ends $\mathbb{R}_{+} \times Y \subset \hat{W}$ and $\mathbb{R}_{-} \times Y \subset \hat{Z}$ with a neck $[-T, T] \times Y$. Let $X^{(T)}$ be equipped with the GM-pair inherited from those of $\hat{W}, \hat{Z}$.

Now fix $\alpha, \beta \in \mathcal{R}^{*}(Y, m+1)$ of the same index and define the parametrized moduli spaces $\mathcal{M}_{\gamma}$ and $\mathcal{M}$ as in the proof of Lemma 6. Let $\ell$ be as in (22). Note that if $\left\{\left(\omega_{n}, T_{n}\right)\right\}$ is any chain-convergent sequence in $\mathcal{M}$ with $\ell\left(\omega_{n}\right) \rightarrow 0$ then $T_{n} \rightarrow \infty$ and the chain-limit is made up of the reducible monopole over $\hat{W}$ and one element from each of the sets $\check{M}\left(\alpha, \theta_{m+1}\right)$ and $M\left(\hat{Z} ; \theta_{m}, \beta\right)$ (which must then both be finite). We now copy the construction of $\hat{\sigma}$ in the proof of Lemma 5, with $T$ and $M\left(\hat{Z} ; \theta_{m}, \beta\right)$ playing the roles of $\tau_{2}$ and $\check{M}\left(\mathbb{R} \times Y ; \theta_{m}, \beta\right)$, resp. Let $\mathcal{M}^{\prime} \subset \mathcal{M}$ be the zero-set of $\hat{\sigma}$ and set

$$
\mathcal{M}^{\prime \prime}:=\mathcal{M}^{\prime}+\mathcal{M}_{z}
$$

By considering the boundary points and ends of $\mathcal{M}^{\prime \prime}$ one finds that

$$
J Q=B+\hat{\delta}^{\prime} \delta+d f \pm f d,
$$

where $J: \mathrm{CF}^{*}(Y, m) \rightarrow \mathrm{CF}^{*}(Y, m+1)$ is a cochain map which induces the canonical map in cohomology, $B$ is of the kind discussed in Lemma 6, and

$$
\hat{\delta}^{\prime}(1)=\sum_{\gamma}\left(\# M\left(\hat{Z} ; \theta_{m}, \gamma\right)\right) \gamma \in \mathrm{CF}^{2 m}(Y, m+1) .
$$

Combining equation (24) with Lemma 6] we conclude that $\bar{J} \bar{Q}=$ id. This completes the proof of Lemma 7, and also of Proposition [12.

Proposition 13 Let $\ell$ be any natural number. Then the kernel and image of the natural homomorphism

$$
H F^{*}(Y, G ; m) \rightarrow H F^{*}(Y, G ; m+\ell)
$$

are

$$
\sum_{j=0}^{\ell-1} \operatorname{im}\left(\delta_{j}^{\prime}\right) \quad \text { and } \quad \bigcap_{j=0}^{\ell-1} \operatorname{ker}\left(\delta_{j}\right)
$$

respectively. 
Proof. For both the kernel and the image, one inclusion follows from Proposition [1](ii), whereas the other inclusion is easily proved by induction on $\ell$ using Propositions 11, 12] and the functoriality of the homomorphisms in question with respect to composition of cobordisms.

We will now define the maps $D, D^{\prime}$ in the sequence (1) and prove that the sequence is exact. Fix $m \in \mathfrak{m}(Y)$ and let

$$
\begin{gathered}
v: \underline{\mathrm{HF}}^{2 m+1}(Y ; G) \stackrel{\approx}{\longrightarrow} \mathrm{HF}^{2 m+1}(Y, m ; G), \\
w: \mathrm{HF}^{2 m}(Y, m+1 ; G) \stackrel{\approx}{\longrightarrow} \overline{\mathrm{HF}}^{2 m}(Y ; G)
\end{gathered}
$$

be the canonical maps. For $z \in \overline{\mathrm{HF}}^{2 m}(Y ; G)$ and $g \in G$ we define

$$
D(z):=x^{m} \otimes \delta_{0} w^{-1}(z), \quad D^{\prime}\left(x^{m} \otimes g\right):=v^{-1} \delta_{0}^{\prime}(g) .
$$

We set $D=0$ on $\overline{\mathrm{HF}}^{2 m+1}(Y ; G)$. (There is little choice, since $P^{2 m+1}(Y)=$ 0.)

From Proposition 11 we deduce that $D, D^{\prime}$ are $\mathbb{Z}[u]-$ homomorphisms and that the two right-most squares of Diagram (2) commute.

Proposition 14 The fundamental sequence (1) is exact.

Proof. Exactness at the terms $\underline{\mathrm{HF}}$ and $\overline{\mathrm{HF}}$ follows from Proposition 13 , and Lemma 5 yields $D^{\prime} D=0$. It remains to show that $\operatorname{ker}\left(D^{\prime}\right) \subset \operatorname{im}(D)$. Suppose $D^{\prime}\left(x^{m} \otimes g\right)=0$. This means there is a chain $\sigma=\sum_{j} \alpha_{j} \otimes g_{j}$ with $\alpha_{j} \in \mathcal{R}(Y, m)$ and $g_{j} \in G$ such that

$$
\rho \otimes g+d \sigma=0
$$

where $\rho=\delta^{\prime}(1) \in \mathrm{CF}^{2 m+1}(Y, m)$. The oriented 1 -manifold $M\left(\mathbb{R} \times Y ; \theta_{m}, \theta_{m+1}\right)$ has (generically) exactly one boundary point (namely the reducible one). Counting with sign we have that the number of boundary points plus the number of ends of that moduli space must be zero, and similarly the number of ends of $M\left(\mathbb{R} \times Y ; \alpha_{j}, \theta_{m+1}\right)$ is zero. Thus

$$
\pm 1=\langle\rho, \phi\rangle+\delta \psi, \quad 0=\left\langle d \alpha_{j}, \phi\right\rangle+\delta \psi_{j}
$$

for a suitable chain $\phi \in \mathrm{CF}_{2 m+1}(Y, m)$ and cochains $\psi, \psi_{j} \in \mathrm{CF}^{2 m}(Y, m+1)$. Tensoring the second equation with $g_{j}$, summing over $j$, and adding the first equation tensored with $g$ we obtain $\pm g=\delta \tau$, where

$$
\tau=\psi \otimes g+\sum_{j} \psi_{j} \otimes g_{j}
$$

A similar argument shows that $d \tau=0$, hence $x^{m} \otimes g \in \operatorname{im}(D)$. 


\section{Reduced Floer groups}

In this section we will show how the reduced Floer cohomology $\widehat{\mathrm{HF}}^{*}(Y ; G)$ can be computed in an arbitrary chamber $m$ by factoring out interaction in $\operatorname{HF}^{*}(Y, m ; G)$ with the reducible critical point. We will then prove Theorem 7 .

Definition 3 We define the reduced Floer cohomology group $\widehat{H F}^{*}(Y, m ; G)$ by

$$
\widehat{H F}^{q}(Y, m ; G)=Z^{q} / B^{q},
$$

where

$$
Z^{*}=\bigcap_{j \geq 0} \operatorname{ker}\left(\delta_{j}\right), \quad B^{*}=\sum_{j \geq 0} \operatorname{im}\left(\delta_{j}^{\prime}\right)
$$

We define

$$
u: \widehat{\mathrm{HF}}^{*}(Y, m ; G) \rightarrow \widehat{\mathrm{HF}}^{*+2}(Y, m ; G)
$$

as follows: Choose a map $v$ as in Proposition 8 . Let $Z^{\prime *}\left(\operatorname{resp} . B^{\prime *}\right)$ be the set of cocycles in $\mathrm{CF}^{*}(Y, m) \otimes G$ representing an element of $Z^{*}$ (resp. $B^{*}$ ). By Proposition 8 the endomorphism $v \otimes 1$ maps each of $Z^{*}$ and $B^{*}$ into itself and therefore induces the desired endomorphism of $Z^{*} / B^{\prime *}=\widehat{\mathrm{HF}}^{*}(Y, m ; G)$.

Let $W$ be a cobordism as in Section 8 . If the assumptions of either part (i) or part (ii) of Proposition [11 are satisfied then by Propositions 9 , 11] the map $W^{*}$ gives rise to a homomorphism

$$
\widehat{\mathrm{HF}}^{*}\left(Y_{1}, m_{1} ; G\right) \rightarrow \widehat{\mathrm{HF}}^{*-d}\left(Y_{2}, m_{2} ; G\right)
$$

which commutes with the $u$-maps.

Proposition 15 (i) If $m_{1} \leq m_{2}$ then the spinc cobordism $[0,1] \times Y$ induces an isomorphism

$$
\widehat{H F}^{*}\left(Y, m_{1} ; G\right) \stackrel{\approx}{\rightarrow} \widehat{H F}^{*}\left(Y, m_{2} ; G\right) .
$$

(ii) $\widehat{H F}^{*}(Y, m ; G)$ is canonically isomorphic to $\widehat{H F}^{*}(Y ; G)$ as a $\mathbb{Z}[u]$ module.

Proof. Statement (i) is an immediate consequence of Propositions 11 (ii) and 13. To prove (ii), recall that if $2 m_{1} \leq q \leq 2 m_{2}-1$ then by Proposition 6 we have a sequence of homomorphisms

$$
{\underline{\mathrm{HF}^{q}}}^{\stackrel{\approx}{\rightarrow}} \mathrm{HF}_{m_{1}}^{q} \rightarrow \mathrm{HF}_{m_{2}}^{q} \stackrel{\approx}{\rightarrow} \overline{\mathrm{HF}}^{q}
$$


where we have used the same abbreviations as in (14). By Proposition 13 the image of the middle homomorphism is precisely $\widehat{\mathrm{HF}}^{q}\left(Y, m_{2} ; G\right)$. On the other hand, that image can clearly be identified with $\widehat{\mathrm{HF}}^{*}(Y ; G)$.

Proof of Theorem 7; Choose $m \in \mathfrak{m}(Y)$ and a sequence $\left\{s_{j}\right\}$ of generic sections of the natural complex line bundle over $\mathcal{B}^{*}(W)$. For any $\alpha, \beta \in$ $\mathcal{R}^{*}(Y, m)$ and non-negative integer $k$ let

$$
M_{\alpha \beta k}:=\left\{\omega \in M(\hat{W} ; \alpha, \beta): s_{j}\left(\left.\omega\right|_{W}\right)=0, \quad j=1, \ldots, k\right\} .
$$

Define a cochain map

$$
P_{k}: \mathrm{CF}^{*}(Y, m) \rightarrow \mathrm{CF}^{*-2 n+2 k}(Y, m)
$$

on generators by

$$
P_{k}(\alpha)=\sum_{\beta}\left(\# M_{\alpha \beta k}\right) \beta .
$$

Let $\psi_{k}$ denote the induced endomorphism of $\operatorname{HF}^{*}(Y, m)$ (with integral coefficients). In analogy with Proposition 11 (i) one has

$$
\delta_{j} \psi_{k}=0, \quad \psi_{k} \delta_{j}^{\prime}=0
$$

for all $j \geq 0$, hence $\psi_{k}$ gives rise to an endomorphism $\hat{\psi}_{k}$ of $\widehat{\mathrm{HF}}^{*}(Y, m)$. Choose a map $v$ as in Proposition 8. Then

$$
P_{k+1}-v P_{k}+\delta^{\prime} \delta_{(k)}=d \phi_{k} \pm \phi_{k} d,
$$

where $\delta_{(k)}$ and $\phi_{k}$ are maps similar to $\delta_{W}$ and $\phi$ in Proposition 9. By induction on $k$ we conclude that $\hat{\psi}_{k}=u^{k} \hat{\psi}_{0}$ for all $k$. By definition, $\hat{\psi}_{0}=$ $\hat{\psi}(W)$. Now

$$
\mathrm{SW}(X)=L\left(P_{n}\right)=L\left(\psi_{n}\right)=L\left(\left.\psi_{n}\right|_{Z^{*}}\right)=L\left(\hat{\psi}_{n}\right)=L\left(u^{n} \hat{\psi}(W)\right) .
$$

The first equality is basic gluing theory, the signs being given by Proposition 3. The second equality is the general fact that the Lefschetz number of a degree 0 chain endomorphism of a finitely generated chain complex agrees with the Lefschetz number of the induced endomorphism on homology. The third and fourth equalities hold because $\psi_{n}$ takes values in $Z^{*}$ and vanishes on $B^{*}$. 


\section{The $h$-invariant}

In this section we will work with Floer cohomology with coefficients in a fixed field $\mathbb{F}$, which will usually be suppressed from notation. $Y$ will denote a $\operatorname{spin}^{c}$ rational homology 3 -sphere. All 4-manifolds will be smooth.

Proposition 16 Let $m \in \mathfrak{m}(Y)$. Then for the group $H F^{*}(Y, m)$, either $\delta_{0}=0$ or $\delta_{0}^{\prime}=0$.

Proof. This follows immediately from Proposition 8.

Definition 4 For any $m \in \mathfrak{m}(Y)$ set

$$
\zeta=\zeta(Y, m):=\chi\left(H F^{*}(Y, m)\right)-\chi\left(\widehat{H F}^{*}(Y)\right),
$$

where $\chi$ means the Euler characteristic with respect to the mod 2 grading. In other words:

- If $\delta_{0}, \delta_{0}^{\prime}$ are both zero then $\zeta=0$.

- If $\delta_{0} \neq 0$ then $\zeta=\max \left\{n \geq 1: \delta_{n-1} \neq 0\right\}$.

- If $\delta_{0}^{\prime} \neq 0$ then $\zeta=-\max \left\{n \geq 1: \delta_{n-1}^{\prime} \neq 0\right\}$.

Lemma $8 \zeta(-Y,-m)=-\zeta(Y, m)$.

Proof. The vector space $\operatorname{HF}^{*}(-Y,-m)$ is the dual of $\operatorname{HF}_{*}(-Y,-m)=$ $\operatorname{HF}^{-1-*}(Y, m)$. Moreover, the $u-$ map on the former vector space (in the degrees in which it is defined) is the dual of the $u$-map on the latter. Similarly, $\delta_{0}: \mathrm{HF}^{-2 m-2}(-Y,-m) \rightarrow \mathbb{F}$ is the dual of $\delta_{0}^{\prime}: \mathbb{F} \rightarrow \operatorname{HF}^{2 m+1}(Y, m)$. Together with Proposition 10, this proves the lemma.

Proposition 17 Let $W$ be a compact, connected spin ${ }^{c}$ 4-manifold whose boundary is a disjoint union of rational homology spheres $Y_{1}, \ldots, Y_{r}$, where $r \geq 1$, and let $m_{j} \in \mathfrak{m}\left(Y_{j}\right)$. Suppose $b^{+}(W)=0$ and $\zeta\left(Y_{j}, m_{j}\right) \leq 0$ for $j>1$. Then

$$
\zeta\left(Y_{1}, m_{1}\right) \geq \sum_{j=1}^{r} m_{j}+\frac{1}{8}\left(c_{1}\left(\mathcal{L}_{W}\right)^{2}-\sigma(W)\right)
$$

provided the right hand side of the inequality is positive. 
Proof. Let $\gamma_{1}, \ldots, \gamma_{\ell}$ be a collection of disjoint loops in $W$ representing a basis for $H_{1}(W ; \mathbb{Z}) /$ torsion, and let $W^{\prime}$ denote the manifold obtained by performing surgery on each $\gamma_{j}$. Then $b_{1}\left(W^{\prime}\right)=0$. Let $V_{i}$ be a small open tubular neighbourhood of $\gamma_{i}$, and set $W^{-}=W \backslash \cup_{i} V_{i}$. Then with integral coefficients there are isomorphisms

$$
H^{2}(W) \stackrel{\approx}{\rightarrow} H^{2}\left(W^{-}\right) \underset{\leftarrow}{\approx} H^{2}\left(W^{\prime}\right),
$$

and similarly for $H_{2}$. Hence, $W$ and $W^{\prime}$ have the same intersection forms, the induced $\operatorname{spin}^{c}$ structure on $W^{-}$has a unique extension to $W^{\prime}$, and the canonical classes for $W$ and $W^{\prime}$ have the same square. Now let $X:=\hat{W}$ be the result of adding half-infinite tubes to $W$.

Set

$$
\vec{\theta}:=\left(\theta_{m_{1}}, \ldots, \theta_{m_{r}}\right)
$$

Then

$$
\operatorname{dim} M(X ; \vec{\theta})=2 k+1
$$

where

$$
k+1=\operatorname{ind}_{\mathbb{C}}\left(D_{A_{o}}\right)=\sum_{j} m_{j}+\frac{1}{8}\left(c_{1}\left(\mathcal{L}_{W}\right)^{2}-\sigma(W)\right) .
$$

Here $A_{o}$ can be taken to be the reference $\operatorname{spin}^{c}$ connection over $X$ used in defining the moduli space $M(X ; \vec{\theta})$, see [9, Section 3.4].

Suppose $k \geq 0$. By Proposition 1 we may assume that $M(X ; \vec{\theta})$ contains a unique reducible point, and that this point is regular. Let $M^{-}(X ; \vec{\theta})$ be the result of removing from $M(X ; \vec{\theta})$ a small neighbourhood of the reducible point, so that the boundary of $M^{-}(X ; \vec{\theta})$ is diffeomorphic to $\mathbb{C P}^{k}$.

Let $\mathbb{L} \rightarrow \mathcal{B}^{*}(W)$ be the natural complex line bundle. Choose a generic smooth section $s$ of $\oplus^{k} \mathbb{L}$. For any moduli space $M$ over $X$ and subset $\Sigma$ of the irreducible part of $M$ set

$$
c_{1}(\mathbb{L})^{k} \cap \Sigma:=\left\{\omega \in \Sigma: s\left(\left.\omega\right|_{W}\right)=0\right\} .
$$

For $j>1$ the assumption $\zeta\left(Y_{j}, m_{j}\right) \leq 0$ means, by the universal coefficient theorem, that the cycle $\delta \in \mathrm{CF}_{2 m_{j}-2}\left(Y_{j}, m_{j}\right)$ is in fact a boundary, i.e. there is a $\rho_{j} \in \mathrm{CF}_{2 m_{j}-1}\left(Y_{j}, m_{j}\right)$ with

$$
\delta+\partial \rho_{j}=0
$$

where $\partial$ is the boundary operator of the chain complex $\mathrm{CF}_{*}$. For any $\alpha \in$ $\mathcal{R}_{Y_{1}}$ set

$$
M_{\alpha}:=M\left(X ; \alpha, \sigma_{2}, \ldots, \sigma_{r}\right),
$$


where $\sigma_{j}=\rho_{j}+\theta_{m_{j}}$. Here we treat $M(X ; \cdot)$ as a multilinear function, so that $M_{\alpha}$ is a formal linear combination of moduli spaces over $X$. In the case $\alpha=\theta:=\theta_{m_{1}}$ one of the terms appearing in that formal linear combination is $M(X ; \vec{\theta})$. Let $M_{\theta}^{-}$be obtained from $M_{\theta}$ by replacing the term $M(X ; \vec{\theta})$ with $M^{-}(X ; \vec{\theta})$.

For any irreducible $\alpha$ set

$$
\hat{M}_{\alpha}:=c_{1}(\mathbb{L})^{k} \cap M_{\alpha}, \quad \hat{M}_{\theta}^{-}:=c_{1}(\mathbb{L})^{k} \cap M_{\theta}^{-},
$$

where $c_{1}(\mathbb{L})^{k} \cap$. is treated as a linear function. For the formal linear combination $\hat{M}_{\theta}^{-}$of oriented 1-manifolds the number of boundary points plus the number of ends (both counted with sign) must be zero. The number of boundary points is

$$
\#\left(c_{1}(\mathbb{L})^{k} \cap \partial M^{-}(X ; \vec{\theta})\right)= \pm 1 .
$$

The ends of $\hat{M}_{\theta}^{-}$correspond to factorizations over the ends of $X$. Because of the choice of $\sigma_{j}$, only factorizations over the end $\mathbb{R}_{+} \times Y_{1}$ contribute. Therefore, the number of ends of $\hat{M}_{\theta}^{-}$is $\delta a$, where the cocycle $a$ is defined by

$$
a=\sum_{\alpha}\left(\# \hat{M}_{\alpha}\right) \cdot \alpha
$$

the sum being taken over all $\alpha \in \mathcal{R}_{Y_{1}}^{*}$ of index $2 m_{1}-2$. Writing [ ] for cohomology classes we have $[a]=u^{k}[b]$, where

$$
b=\sum_{\beta}\left(\# M_{\beta}\right) \cdot \beta \text {. }
$$

Thus,

$$
\delta_{0} u^{k}[b]=\delta \alpha=\mp 1,
$$

proving that $\zeta\left(Y_{1}, m_{1}\right) \geq k+1$ as claimed.

Corollary 2 Let $W$ be a compact, connected $\operatorname{spin}^{c}$ 4-manifold whose boundary is a disjoint union of rational homology spheres $Y_{1}, \ldots, Y_{r}$, and let $m_{j} \in \mathfrak{m}\left(Y_{j}\right)$. Suppose $b_{2}(W)=0$ and $\zeta\left(Y_{j}, m_{j}\right)=0$ for all $j$. Then $\sum_{j=1}^{r} m_{j}=0$.

Proof. If $\sum_{j} m_{j} \neq 0$ then after perhaps reversing the orientation of $W$ we may assume $\sum_{j} m_{j}>0$, contradicting the proposition.

Lemma $9 \zeta\left(S^{3}, m\right)=m$ for all $m \in \mathbb{Z}$. 
Proof. Since $\zeta\left(S^{3},-m\right)=-\zeta\left(S^{3}, m\right)$, we may assume $m \geq 0$. The lemma clearly holds for $m=0$, because $\operatorname{HF}^{*}\left(S^{3}, 0\right)=0$. Now let $m \geq 1$. Applying Proposition [17 with $W=[0,1] \times S^{3}, m_{1}=m, m_{2}=0$ we obtain $\zeta\left(S^{3}, m\right) \geq m$. On the other hand, Proposition 7 gives $\zeta\left(S^{3}, m\right) \leq m$. Together this proves the lemma.

Proposition 18 Let $W$ be a spin ${ }^{c}$, compact, connected 4-manifold with boundary components $-Y_{0},-Y_{1}, Y_{2}$, such that each $Y_{j}$ is a rational homology sphere and $b_{2}(W)=0$. Suppose $m_{j} \in \mathfrak{m}\left(Y_{j}\right), j=0,1,2$ satisfy $-m_{0}-m_{1}+$ $m_{2}=0$. Then the following hold:

(i) If $\zeta\left(Y_{j}, m_{j}\right)>0$ for $j=0,1$ then

$$
\zeta\left(Y_{2}, m_{2}\right) \geq \zeta\left(Y_{0}, m_{0}\right)+\zeta\left(Y_{1}, m_{1}\right) .
$$

(ii) If $\zeta\left(Y_{0}, m_{0}\right)=0$ then

$$
\zeta\left(Y_{1}, m_{1}\right)=\zeta\left(Y_{2}, m_{2}\right)
$$

Proof. The proofs are similar to those of [8, Lemma 6] and [8, Theorem 14], respectively.

Corollary 3 If $\zeta\left(Y_{1}, m_{1}\right)=\zeta\left(Y_{2}, m_{2}\right) \neq 0$ then $\zeta\left(Y_{1} \#\left(-Y_{2}\right), m_{1}-m_{2}\right)=0$.

Proof. Apply part (i) of the proposition to $W$ or $-W$, where $W$ is the standard cobordism from $Y_{1} \cup\left(-Y_{2}\right)$ to $Y_{1} \#\left(-Y_{2}\right)$.

Proposition 19 For any $Y$ there exists an $m \in \mathfrak{m}(Y)$ such that $\zeta(Y, m)=$ 0 .

Proof. Pick any $m^{\prime} \in \mathfrak{m}(Y)$ and set $k:=\zeta\left(Y, m^{\prime}\right)$. If $k=0$ then we are done. Otherwise, set $m=m^{\prime}-k$. Then Lemma 9 and Corollary 3 give

$$
\zeta(Y, m)=\zeta\left(Y \#\left(-S^{3}\right), m^{\prime}-k\right)=0 .
$$

Proposition 20 If $\zeta(Y, m)=0$ then $\zeta(Y, m+k)=k$ for all integers $k$.

Proof. Let $D \subset(0,1) \times Y$ be an embedded closed 4-ball. Applying Proposition 18 (ii) to $W=([0,1] \times Y) \backslash \operatorname{int}(D)$ we get

$$
\zeta(Y, m+k)=\zeta\left(S^{3}, k\right)=k .
$$


Definition 5 For any spin ${ }^{c}$ rational homology 3-sphere $Y$ we define $h(Y)=$ $h(Y ; \mathbb{F})$ to be the unique $m \in \mathfrak{m}(Y)$ such that $\zeta(Y, m)=0$.

It follows from Propositions 19 and 20 that $h(Y)$ is well defined, and that

$$
h(Y)=m-\zeta(Y, m)
$$

for all $m \in \mathfrak{m}(Y)$.

Theorem 1 is now an immediate consequence of Propositions 13, 19, and 20. and Theorem 2 follows from (25).

Proof of Theorem 3: Set $m_{j}:=h\left(Y_{j}\right)$ for $j=1,2$ and $m_{3}:=h\left(Y_{1} \# Y_{2}\right)$. Applying Corollary 2 to the standard cobordism $W$ from $Y_{1} \cup Y_{2}$ to $Y_{1} \# Y_{2}$ we obtain $-m_{1}-m_{2}+m_{3}=0$, which is the assertion of the theorem.

Proof of Theorem 4: Set $m_{j}:=h\left(Y_{j}\right)$ for $j>1$ and choose $m_{1} \in \mathfrak{m}\left(Y_{1}\right)$ with

$$
\sum_{j=1}^{r} m_{j}+\frac{1}{8}\left(c_{1}\left(\mathcal{L}_{W}\right)^{2}-\sigma(W)\right)>0 .
$$

The theorem then follows from Proposition 17.

We now address the dependence of $h$ on the coefficient field $\mathbb{F}$. Let $\mathbb{F}$ have characteristic $p$ and let $\mathbb{K}$ be the prime field of $\mathbb{F}$, i.e. $\mathbb{K}=\mathbb{Q}$ if $p=0$ and $\mathbb{K}=\mathbb{Z} / p \mathbb{Z}$ if $p>0$. Then

$$
\operatorname{HF}^{*}(Y ; \mathbb{F})=\operatorname{HF}^{*}(Y ; \mathbb{K}) \otimes_{\mathbb{K}} \mathbb{F}
$$

from which one easily deduces that

$$
h(Y ; \mathbb{F})=h(Y ; \mathbb{K}) .
$$

Thus we may write $h_{p}(Y)$ instead of $h(Y ; \mathbb{F})$.

Note that if $Y$ admits a metric $g$ of positive scalar curvature then $(g, 0)$ is an ND-pair for $Y$ for which the corresponding Floer cochain complex vanishes. Hence $h_{p}(Y)=I(g, 0)$ for all $p$. (The index $I$ was defined in (44)).

If $h_{p}(Y) \neq h_{0}(Y)$ for some $p$ then it is not hard to see that $\operatorname{HF}^{*}\left(Y, h_{0}(Y) ; \mathbb{Z}\right)$ contains an element of order $p$ (in degree $2 h_{0}(Y) \pm 1$ ). The following proposition shows that this group is in a sense the universal home for torsion:

Proposition 21 For any $m, q$ let $T_{m}^{q}$ be the torsion subgroup of $H F^{q}(Y, m ; \mathbb{Z})$. For $m_{1} \leq m_{2}$ the canonical homomorphism

$$
H F^{q}\left(Y, m_{1} ; \mathbb{Z}\right) \rightarrow H F^{q}\left(Y, m_{2} ; \mathbb{Z}\right)
$$


restricts to a homomorphism

$$
T_{m_{1}}^{q} \rightarrow T_{m_{2}}^{q}
$$

which is injective if $m_{2} \leq h_{0}(Y)$ and surjective if $h_{0}(Y) \leq m_{1}$.

Proof. The element $\delta_{j}^{\prime} \in \operatorname{HF}^{*}\left(Y, m_{1} ; \mathbb{Z}\right)$ has infinite order for $j=0, \ldots, h_{0}(Y)-$ $m_{1}-1$ if $m_{2} \leq h_{0}(Y)$ and finite order for all $j$ if $h_{0}(Y) \leq m_{1}$. The proposition now follows from Proposition 13 .

\section{Proof of Theorem 8}

Lemma 10 Let $A, B$ be $r \times r$ complex matrices and $m$ a natural number such that $\operatorname{tr}\left(A^{n}\right)=\operatorname{tr}\left(B^{n}\right)$ for all natural numbers $n$ satisfying $m \leq n<2 r+$ $m$. Then $A$ and $B$ have the same characteristic polynomial. In particular, $\operatorname{tr}(A)=\operatorname{tr}(B)$.

Proof. Let $E$ denote the set of all the eigenvalues of $A$ and $B$. For any square matrix $C$ and complex number $\lambda$ let $m_{C}(\lambda)$ denote the multiplicity of $\lambda$ as an eigenvalue of $C$. It suffices to show that $m_{A}(\lambda)=m_{B}(\lambda)$ for all $\lambda \neq 0$. So suppose $m_{A}(\lambda)>0$ and consider the polynomial

$$
P(z)=z^{m} \prod_{a \in E \backslash\{\lambda\}}(z-a) .
$$

Putting $A$ in triangular form we see that the multiplicity of $P(\lambda)$ as an eigenvalue of $P(A)$ is $m_{A}(\lambda)$, and there are no other non-zero eigenvalues. Similarly for $B$. Hence

$$
m_{A}(\lambda) \cdot P(\lambda)=\operatorname{tr}(P(A))=\operatorname{tr}(P(B))=m_{B}(\lambda) \cdot P(\lambda) .
$$

But $P(\lambda) \neq 0$, so $m_{A}(\lambda)=m_{B}(\lambda)$.

Corollary 4 Let $U=U_{0} \oplus U_{1}$ and $V=V_{0} \oplus V_{1}$ be finite-dimensional mod 2 graded complex vector spaces. Let $f=f_{0} \oplus f_{1} \in \operatorname{End}(U)$ and $g=g_{0} \oplus g_{1} \in$ $\operatorname{End}(V)$ preserve degrees. If $m$ is a natural number such that the Lefschetz numbers $L\left(f^{n}\right)=L\left(g^{n}\right)$ for all $n \geq m$, then $L(f)=L(g)$.

Proof. Apply the lemma to $f_{0} \oplus g_{1}$ and $g_{0} \oplus f_{1}$.

Proof of Theorem 8: Because $Y_{j}$ is non-separating, there is a loop in $X$ whose intersection number with $Y_{j}$ is \pm 1 . Hence each of $Y_{0}$ and $Y_{1}$ represents a generator of $H_{3}(X ; \mathbb{Z})$, and by assumption these two generators are equal. 
Let $f_{j}: X \rightarrow S^{1}$ be a smooth map such that 1 is a regular value of $f_{j}$ and $\left(f_{j}\right)^{-1}(1)=Y_{j}$ as oriented manifolds. Then $f_{0}$ and $f_{1}$ represent the same generator of $\left[X, S^{1}\right] \approx H^{1}(X ; \mathbb{Z})$. Set

$$
X_{j, \infty}=\left\{(x, t) \in X \times \mathbb{R}: f_{j}(x)=e^{2 \pi i t}\right\} .
$$

Then the projection

$$
\pi_{j, \infty}: X_{j, \infty} \rightarrow X
$$

is a covering whose group of deck transformations is canonically isomorphic to $\mathbb{Z}$. Let

$$
q_{j}: X_{j, \infty} \rightarrow \mathbb{R}
$$

be the projection onto the second factor. We may take

$$
W_{j}:=\left(q_{j}\right)^{-1}[0,1]
$$

as the definition of $W_{j}$. Then

$$
W_{j, n}:=\left(q_{j}\right)^{-1}[-\lfloor n / 2\rfloor, n-\lfloor n / 2\rfloor]
$$

is the result of gluing together $n$ copies of $W_{j}$ in a chain.

Since $f_{0}$ and $f_{1}$ are homotopic, the coverings $\pi_{0, \infty}$ and $\pi_{1, \infty}$ are isomorphic. In particular, $\pi_{0, \infty}$ restricts to a trivial covering of $Y_{1}$. Let $B$ be a component of $\left(\pi_{0, \infty}\right)^{-1}\left(Y_{1}\right)$. Since $B$ is compact, there is a natural number $m$ such that $B$ is contained in the interior of $W_{0, m}$. For every natural number $n$ set $X_{n}:=X_{0, \infty} / n \mathbb{Z}$ and let

$$
\pi_{n}: X_{n} \rightarrow X
$$

be the natural $n$-fold covering. For $n \geq m$ there is a component $C_{j}$ of $\pi_{n}^{-1}\left(Y_{j}\right), j=0,1$, with $C_{0} \cap C_{1}=\emptyset$. Let $C_{j}$ be oriented such that the diffeomorphism $C_{j} \rightarrow Y_{j}$ obtained by restriction of $\pi_{n}$ is orientation preserving. Then $X_{n}$ is the union of two compact, connected, codimension 0 submanifolds $Z_{0}, Z_{1}$ with

$$
Z_{0} \cap Z_{1}=C_{0} \cup C_{1}, \quad \partial Z_{j}=\left(-C_{j}\right) \cup C_{1-j} .
$$

Moreover, $b_{1}\left(Z_{j}\right)=0=b^{+}\left(Z_{j}\right)$. If each $Y_{j}$ is an integral homology sphere then it follows from Theorem 4 and Elkies' theorem that $h_{p}\left(Y_{0}\right)=h_{p}\left(Y_{1}\right)$. The same conclusion holds if $b_{2}(X)=0$, because then $b_{2}\left(Z_{j}\right)=0$, too.

Returning to the general case, we can identify

$$
W_{j, n}=Z_{1-j} \cup_{C_{1-j}} Z_{j}
$$


Setting

$$
A_{j}:=\hat{\psi}\left(W_{j}\right): \widehat{\mathrm{HF}}^{*}\left(Y_{j}\right) \rightarrow \widehat{\mathrm{HF}}^{*}\left(Y_{j}\right)
$$

we have

$$
\left(A_{j}\right)^{n}=\hat{\psi}\left(W_{j, n}\right)=\hat{\psi}\left(Z_{1-j}\right) \circ \hat{\psi}\left(Z_{j}\right) .
$$

Since $\hat{\psi}\left(Z_{j}\right)$ preserves the mod 2 grading, we conclude that

$$
L\left(\left(A_{0}\right)^{n}\right)=L\left(\left(A_{1}\right)^{n}\right) \quad \text { for all } n \geq m .
$$

Corollary 4 now says that $L\left(A_{0}\right)=L\left(A_{1}\right)$, and the theorem is proved.

\section{A finite-dimensional analogue}

Whereas Marcolli-Wang's construction of equivariant Seiberg-Witten Floer homology [15] was modelled on Austin-Braam's equivariant Morse complex [1, it may seem less obvious why the groups $\overline{\mathrm{HF}}^{*}(Y)$ deserve being called equivariant Floer cohomology. This section will provide justification for this name by means of a finite-dimensional analogue. We will use integer coefficients, for simplicity.

Conceptually, a natural setting for our construction of Floer cohomology is the Hilbert manifold $\mathcal{B}_{y}(Y)$ of $L_{1}^{2}$ configurations $(B, \Psi)$ on $Y$ modulo $L_{2}^{2}$ gauge transformations $u: Y \rightarrow \mathrm{U}(1)$ with $u(y)=1$, where $y \in Y$ is a basepoint. There is a smooth action of $\mathrm{U}(1)$ on $\mathcal{B}_{y}(Y)$ whose fixed-points are the elements $[B, \Psi]$ with spinor $\Psi=0$. Away from the fixed-point set $F$ the action is free, so that the quotient

$$
\mathcal{B}^{*}(Y)=\left(\mathcal{B}_{y}(Y) \backslash F\right) / \mathrm{U}(1)
$$

is also a Hilbert manifold. The Chern-Simons-Dirac functional on $Y$ descends to a smooth $\mathrm{U}(1)$-invariant function

$$
\underline{\vartheta}: \mathcal{B}_{y}(Y) \rightarrow \mathbb{R}
$$

which in turn induces a smooth function

$$
\underline{\underline{\vartheta}}: \mathcal{B}^{*}(Y) \rightarrow \mathbb{R}
$$

An important point here is that $\underline{\vartheta}$ has exactly one critical point which is also a fixed-point. The group $\operatorname{HF}^{*}(Y, m)$ was defined as the cohomology of a "Morse complex" of $\underline{\underline{\vartheta}}$, whereas $\overline{\mathrm{HF}}^{q}(Y)$ was obtained from $\operatorname{HF}^{*}(Y, m)$ by a limiting construction as $m=\frac{1}{2} \operatorname{ind}(\theta) \rightarrow \infty$. 
We will now interpret this construction in terms of finite-dimensional Morse theory (see for instance [2]). As our analogue of $\mathcal{B}_{y}(Y)$ we consider a compact manifold-with-boundary $V$ of positive dimension on which $\mathrm{U}(1)$ acts smoothly, and such that the action is free away from one fixed-point $p$ which lies in the interior of $V$. In the proof of Proposition 22 below we will show that the $\mathrm{U}(1)$-action on $V$ around $p$ is equivalent to the standard action on $\mathbb{C}^{n}$ around the origin for some $n$. In particular, $\operatorname{dim} V=2 n$. (It is easy to deduce from this that the boundary $\partial V$ must be non-empty.) We assume $\partial V$ is the disjoint union of two relatively open subsets $V_{0}$ and $V_{1}$, one of which might be empty. Let

$$
\pi: V \rightarrow B:=V / \mathrm{U}(1)
$$

be the projection onto the quotient space and set $\underline{p}:=\pi(p)$ and $B_{i}:=\pi\left(V_{i}\right)$ for $i=0,1$. Then the manifold

$$
B^{*}:=B \backslash\{\underline{p}\} .
$$

is our analogue of $\mathcal{B}^{*}(Y)$. Now let

$$
f: V \rightarrow[0,1]
$$

be a smooth $\mathrm{U}(1)$-invariant function such that the following hold:

- $f^{-1}(i)=V_{i}$ for $i=0,1$.

- $p$ is a non-degenerate critical point of $f$ of maximal index, i.e. $\operatorname{ind}(p)=$ $2 n$. (In particular, $p$ is a local maximum of $f$.)

- The induced function

$$
g: B^{*} \rightarrow \mathbb{R}
$$

has only non-degenerate critical points, all of which lie in the interior of $B^{*}$.

Note that taking ind $(p)$ maximal mirrors the construction of $\overline{\mathrm{HF}}^{*}(Y)$.

Choose a U(1)-invariant Riemannian metric on $V$ and let $B^{*}$ have the Riemannian metric for which

$$
V^{*}:=V \backslash\{p\} \rightarrow B^{*}
$$

is a Riemannian submersion. Then flow-lines of $-\nabla f$ in $V^{*}$ map to flowlines of $-\nabla g$ in $B^{*}$. Let $C$ be the image in $B$ of the set of critical points of $f$. Then

$$
C^{*}:=C \backslash\{\underline{p}\}
$$


is the set of critical points of $g$. For any $a \in C$ let $W^{s}(a)$ and $W^{u}(a)$ denote the stable and unstable manifolds of $a$, resp. (The usual definitions carry over in an obvious way, even though $B$ is not a manifold due to the singular point $\underline{p}$.) In particular, $W^{u}(\underline{p})$ agrees with the image in $B$ of the unstable manifold of $p$ in $V$, and $W^{s}(\underline{p})=\{p\}$. We assume that the Morse-Smale condition holds in the sense that $W^{s}(a)$ and $W^{u}(b)$ intersect transversely for any $a \in C^{*}, b \in C$ (which holds trivially if $b=\underline{p}$ ), and set

$$
M(a, b):=W^{s}(a) \cap W^{u}(b) .
$$

Then

$$
\operatorname{dim} M(a, \underline{p})=2 n-1-\operatorname{ind}(a) .
$$

For comparison: In gauge theory one has, for any $\alpha \in \mathcal{R}_{Y}^{*}$,

$$
\operatorname{dim} M(\alpha, \theta)=2 m-1-\operatorname{ind}(\alpha) .
$$

This means that $2 n=\operatorname{ind}(p)$ corresponds to $2 m=\operatorname{ind}(\theta)$. The analogue of $\operatorname{HF}^{*}(Y, m)$ is now the cohomology of the usual Morse complex of $g$, which is isomorphic to the singular cohomology of the pair $\left(B^{*}, B_{0}\right)$.

Before stating the main result of this section recall that if a Lie group $K$ acts on a space $X$ then the equivariant cohomology $H_{K}^{*}(X)$ is by definition the cohomology of the homotopy quotient

$$
X_{K}:=X \times_{K} E K,
$$

where $E K \rightarrow B K$ is a universal principal $K$-bundle. If $Y$ is a $K$-invariant subspace of $X$ such that $Y_{K} \subset X_{K}$ has the subspace topology (which it always has when $K$ is compact and $Y$ is closed in $X$ ) then we define

$$
H_{K}^{*}(X, Y):=H^{*}\left(X_{K}, Y_{K}\right) .
$$

If the projection $X \rightarrow X / K$ is a principal bundle then the canonical map $X_{K} \rightarrow X / K$ is a weak homotopy equivalence and therefore induces an isomorphism on (co)homology. For instance, this applies to the map

$$
\left(V^{*}\right)_{\mathrm{U}(1)} \rightarrow V^{*} / \mathrm{U}(1)=B^{*}
$$

Therefore, the inclusions of $\left(V^{*}, V_{0}\right)$ and $(\{p\}, \emptyset)$ in $\left(V, V_{0}\right)$ induce homomorphisms

$$
H^{q}\left(B^{*}, B_{0}\right) \leftarrow H_{\mathrm{U}(1)}^{q}\left(V, V_{0}\right) \rightarrow H^{q}\left(\mathbb{C P}^{\infty}\right)
$$

Proposition 22 The first map in (26) is an isomorphism for $q \leq 2 n-1$ whereas the second map is an isomorphism for $q \geq 2 n$. 
Thus, the equivariant cohomology of $\left(V, V_{0}\right)$ (as an Abelian group) is completely determined by the cohomology of $\left(B^{*}, B_{0}\right)$. An analogous statement holds for the Floer groups provided $m$ is so large that $\underline{\operatorname{HF}}^{q}(Y)=0$ for $q \geq 2 m$. Namely, in the sequence

$$
\operatorname{HF}^{q}(Y, m) \rightarrow \overline{\operatorname{HF}}^{q}(Y) \stackrel{D}{\rightarrow} P^{q}(Y)
$$

the first map is an isomorphism for $q \leq 2 m-1$ whereas $D$ is an isomorphism for $q \geq 2 m$. (See Subsection 2.2.) Thus, in this analogy with finitedimensional Morse theory, $\overline{\mathrm{HF}}^{*}(Y)$ plays the role of the $\mathrm{U}(1)$-equivariant cohomology of $\mathcal{B}_{y}(Y)$ (since the latter has no boundary).

Proof of Proposition 22: Set $\mathbb{T}:=\mathrm{U}(1)$. Note that the action of $\mathbb{T}$ on $V$ induces a representation of $\mathbb{T}$ on the tangent space $T_{p} V$. Let $D^{\prime} \subset T_{p} V$ be a small closed ball centred at the origin. Then $\exp : D^{\prime} \rightarrow V$ is a $\mathbb{T}$-equivariant embedding. Because $\mathbb{T}$ acts freely on $V^{*}$ it follows from the classification of real $\mathbb{T}$-representations that for some integer $n$ there is an isomorphism $T_{p} V \stackrel{\widetilde{\rightarrow}}{\rightarrow} \mathbb{C}^{n}$ of euclidean vector spaces which is equivariant with respect to the standard action of $\mathbb{T}$ on $\mathbb{C}^{n}$.

Let $S$ be the boundary of the geodesic ball $D:=\exp \left(D^{\prime}\right)$. Note that there is a deformation retraction of the homotopy quotient $D_{\mathbb{T}}$ onto a subspace which we can identify with $B \mathbb{T}=\mathbb{C P}^{\infty}$. Moreover, the inclusion $S_{\mathbb{T}} \rightarrow D_{\mathbb{T}}$ is homotopic to a classifying map $S_{\mathbb{T}} \rightarrow \mathbb{C P}^{\infty}$ of the $\mathbb{T}$-bundle $S \times E \mathbb{T} \rightarrow S_{\mathbb{T}}$. From the Mayer-Vietoris sequence of the pairs $\left(\left(V^{*}\right)_{\mathbb{T}},\left(V_{0}\right)_{\mathbb{T}}\right)$ and $\left(D_{\mathbb{T}}, \emptyset\right)$ in $\left(V_{\mathbb{T}},\left(V_{0}\right)_{\mathbb{T}}\right)$ we then obtain for every integer $q$ a short exact sequence

$$
0 \longrightarrow H_{\mathbb{T}}^{q}\left(V, V_{0}\right) \longrightarrow H^{q}\left(B^{*}, B_{0}\right) \oplus H^{q}\left(\mathbb{C P}^{\infty}\right) \stackrel{a+b}{\longrightarrow} H^{q}\left(\mathbb{C P}^{n-1}\right) \longrightarrow 0,
$$

where $b$ is an isomorphism for $q \leq 2 n-1$. Since

$$
H^{q}\left(B^{*}, B_{0}\right)=H^{q}\left(\mathbb{C P}^{n-1}\right)=0 \quad \text { for } q \geq 2 n
$$

for dimensional reasons, the proposition follows.

\section{References}

[1] D. M. Austin and P. J. Braam. Morse-Bott theory and equivariant cohomology. In The Floer Memorial Volume, number 133 in Progr. Math., pages 123-183. Birkhäuser, Basel, 1995.

[2] A. Banyaga and D. Hurtubise. Lectures on Morse homology. Number 29 in Kluwer Texts in Mathematical Sciences. Springer, 2004. 
[3] Ch. Bär. Harmonic spinors for twisted Dirac operators. Math. Ann., 309:225-246, 1997.

[4] S. K. Donaldson. Floer Homology Groups in Yang-Mills Theory. Cambridge University Press, 2002.

[5] N. D. Elkies. A characterization of the $\mathbb{Z}^{n}$ lattice. Math. Res. Letters, 2:321-326, 1995.

[6] A. Floer. An instanton invariant for 3-manifolds. Comm. Math. Phys., 118:215-240, 1988.

[7] K. A. Frøyshov. The Seiberg-Witten equations and four-manifolds with boundary. Math. Res. Lett., 3:373-390, 1996.

[8] K. A. Frøyshov. Equivariant aspects of Yang-Mills Floer theory. Topology, 41:525-552, 2002.

[9] K. A. Frøyshov. Compactness and gluing theory for monopoles, volume 15 of Geometry \& Topology Monographs. Geometry \& Topology Publications, 2008.

[10] M. Furuta and H. Ohta. Differentiable structures on punctured 4manifolds. Topology and its Appl., 51:291-301, 1993.

[11] N. Hitchin. Harmonic spinors. Adv. in Math., 14:1-55, 1974.

[12] J. Kazdan. Unique continuation in geometry. Comm. Pure and Applied Math., 41:667-681, 1988.

[13] P. B. Kronheimer and T. S. Mrowka. Monopoles and Three-Manifolds. Cambridge University Press, 2007.

[14] Y. Lim. The equivalence of Seiberg-Witten and Casson invariants for homology 3-spheres. Math. Res. Lett., 6:631-643, 1999.

[15] M. Marcolli and B-L. Wang. Equivariant Seiberg-Witten Floer homology. Comm. Anal. and Geom., 9:451-639, 2001.

[16] Ch. Okonek and A. Teleman. Seiberg-Witten invariants for 4-manifolds with $b_{+}=0$. In Th. Peternell and F.-O. Schreyer, editors, Complex analysis and algebraic geometry, pages 347-357. Walter de Gruyter, 2000 . 
[17] P. S. Ozsváth and Z. Szabó. Absolutely graded Floer homologies and intersection forms for four-manifolds with boundary. Adv. Math., 173:179-261, 2003.

[18] P. S. Ozsváth and Z. Szabó. Holomorphic disks and topological invariants for closed three-manifolds. Ann. Math. (2), 159:1027-1158, 2004.

[19] J. Robbin and D. Salamon. The spectral flow and the Maslov index. Bull. Lond. Math. Soc., 27:1-33, 1995.

[20] D. Ruberman and N. Saveliev. Rohlin's invariant and gauge theory II. Mapping tori. Geometry \& Topology, 8:35-76, 2004.

[21] A. Teleman. Donaldson theory on non-Kählerian surfaces and class VII surfaces with $b_{2}=1$. Invent. Math., 162(3):493-521, 2005.

INSTITUT FÜR MATHEMATIK, UNIVERSITÄT ZÜRICH,

WINTERTHURERSTRASSE 190,

CH-8057 ZÜRICH,

SWITZERLAND

E-mail: froyshov@math.uzh.ch 